\title{
Subnormal operators regarded as generalized observables and compound-system-type normal extension
related to $\mathfrak{s u}(1,1)$
}

\author{
Masahito Hayashi†and Fuminori Sakaguchi† \\ $\dagger$ Department of Mathematics, Graduate School of Science, Kyoto University, Kyoto, \\ 606-8502, Japan. e-mail masahito@kusm.kyoto-u.ac.jp \\ $\ddagger$ Department of Electrical and Electronics Engineering, Fukui University, 3-9-1, \\ Bunkyo, Fukui, 910-8507, Japan. e-mail saka@dignet.fuee.fukui-u.ac.jp
}

\begin{abstract}
In this paper, subnormal operators, not necessarily bounded, are discussed as generalized observables. In order to describe not only the information about the probability distribution of the output data of their measurement but also a framework of their implementations, we introduce a new concept compound-systemtype normal extension, and we derive the compound-system-type normal extension of a subnormal operator, which is defined from an irreducible unitary representation of the algebra $\mathfrak{s u}(1,1)$. The squeezed states are characterized as the eigenvectors of an operator from this viewpoint, and the squeezed states in multi-particle systems are shown to be the eigenvectors of the adjoints of these subnormal operators under a representation. The affine coherent states are discussed in the same context, as well.
\end{abstract}

Submitted to: J. Phys. A: Math. Gen.

PACS numbers: 03.65.Bz, 03.65.Db, 03.65.Fd, 02.20.Qs 


\section{Introduction}

In quantum mechanics, observables are described by self-adjoint operators and the probability distributions of the output data of their measurement are determined by the spectral measures of those self-adjoint operators and the density operators of states.

When a linear operator has its spectral measure, it is a normal operator where its self-adjoint part and its skew-adjoint part commute with each other (Lemma 3). In a broader sense, therefore, it can be regarded as a complexified observable. (NB: From this viewpoint, in the following, we will use the expression "measurement of a normal operator" in this wider sense, even if the normal operator is not always selfadjoint.) However, the measurements in quantum systems, which are not necessarily the measurements of any observables, are described by the positive operator-valued measures (POVM), which are a generalization of spectral measures (Definition 5 and Lemma 4). In this paper, from these viewpoints, we try to treat the observables generalized even for the class of subnormal operators团, which is known as a wider class including the class of normal operators. A subnormal operator is defined as the restriction of the normal operator into a narrower domain. As far as the authors know, such a idea generalizing observables was introduced by Yuen and Lax [1]. The pair of the normal operator and the wider domain is called its normal extension (Definition 2). We can define the POVM of a subnormal operator uniquely in a similar sense that we can define the spectral measure of a normal operator uniquely under some condition. (Lemma 19). By this correspondence, we will formulate the measurements of the subnormal operators which are not necessarily bounded. In this paper, we will not only investigate the POVMs of the subnormal operators but also give some examples of frameworks of their implementations in a physical sense.

There are many cases where the adjoint operator of a subnormal operator has eigenvectors with continuous potency and an over-complete eigenvector system. In these cases, the POVM constructed from the over-complete eigenvector system is just the POVM of the subnormal operator (Lemma 22). Thus the subnormal operator is closely related to eigenvectors with continuous potency and to over-complete function systems, and these relations are important for the discussions on the properties of the subnormal operator. This fact may give us an illusion that the adjoint of any operator with a point spectrum with continuous potency would be a subnormal operator. However, the subnormality is not necessarily guaranteed only by the condition that its adjoint has point a spectrum with continuous potencys.

For example, an implementation of the measurement of a subnormal operator has been already known for an actual system in quantum optics. Let $Q$ and $P$ be the multiplication operator and the $(-i)$-times differential operator on the Hilbert space $L^{2}(\mathbb{R})$. A POVM is constructed from the over-complete eigenvector system of the boson annihilation operator $a_{b}:=\sqrt{1 / 2}(Q+i P)$ (known as the coherent states system). Then

$\ddagger$ The concept of subnormality was introduced by Halmos [2, 3].

$\S$ Its counter examples are given in Lemma 36 and Lemma 39. 
this POVM is just the POVM of the boson creation operator $a_{b}^{*}$ which is a subnormal operator. The measurement of this POVM has been implemented as is shown in the following (see section 3, in detail), and is called the heterodyne measurement; this implementation is performed by the measurement of a normal operator on the compound system between the basic system (i.e. the system of interest where the measurement is originally discussed) and an additional ancillary system prepared appropriately. Note that this operation, of measuring a normal operator on the compound system by preparing an additional ancillary system, gives a kind of normal extension of creation operator $a_{b}^{*}$. But, only giving the definition of the normal extension is not sufficient for discussing such a physical operation. For clarifying such a physical operation, in section 3, we will introduce a new concept compound-system-type normal extension which describes not only the normal extension but also a framework of a physical operation (given in Definition 26).

In section 1 , under the circumstance where an irreducible unitary representation of the algebra $\mathfrak{s u}(1,1)$ is given, we will construct two types of operators which have point spectra with continuous potency, and will investigate what condition guarantees the subnormality of these operators. The coherent states of the algebra $\mathfrak{s u}(1,1)$ introduced by Perelomov [4], will be reinterpreted as the eigenvectors of these operators. Moreover, in section 6, we will derive the compound-system-type normal extensions of these operators when they are subnormal operators.

In section 5.1, from the relationship between the irreducible unitary representations of the algebra $\mathfrak{s u}(1,1)$ and those of the affine group ( $a x+b$ group), we will discuss what subnormal operators are related to the irreducible unitary representations of the affine group. Moreover, we will discuss the correspondence between the eigenvectors of this subnormal operator (or the coherent states of the the algebra $\mathfrak{s u}(1,1)$ ) and the coherent states of the affine group. Hence we will show a relationship between our problem and the irreducible unitary representation of the affine group which is closely related to the continuous wavelet transform.

Next, in section 5.2, from the relationship between the representation of the algebra $\mathfrak{s u}(1,1)$ and the squeezed states, it will be confirmed that the squeezed states can be described as the coherent states of the algebra $\mathfrak{s u}(1,1)$ in our context. In other words, the squeezed states are characterized as the eigenvectors of the operators (with point spectra with continuous potency) which are canonically constructed from an irreducible unitary representations of the algebra $\mathfrak{s u}(1,1)$. However, the adjoints of these operators are not necessarily subnormal operators and are not directly regarded as generalized observables.

We can easily confirm that the squeezed states are the eigenvectors of an operator with a point spectrum with continuous potency as follows; according to Yuen [5], let $b_{\mu, \nu}:=\mu a_{b}+\nu a_{b}^{*}$ with $|\mu|^{2}-|\nu|^{2}=1$, and characterize the squeezed state by the eigenvector $|\alpha ; \mu, \nu\rangle$ of the operator $b_{\mu, \nu}$ associated with the eigenvalue $\alpha \in \mathbb{C}$. In the special cases where $\alpha=0$, the vector $|0 ; \mu, \nu\rangle$ can be obtained by operating the action of the group with the generators $\frac{1}{2} Q^{2},-\frac{1}{2} P^{2}$ and $\frac{1}{2}(P Q+Q P)$ upon the boson vacuum 
vector $|0 ; 1,0\rangle$. The algebra with these generators satisfies the commutation relations of the algebra $\mathfrak{s u}(1,1)$. By operating $Q^{-1}\left(\right.$ or $\left.\left(a_{b}^{*}\right)^{-1}\right)$ upon the characteristic equation $b_{\mu, \nu}|0 ; \mu, \nu\rangle=0$ from the left, we have the characteristic equations

$$
\begin{aligned}
& Q^{-1} P|0 ; \mu, \nu\rangle=i \frac{\mu+\nu}{\mu-\nu}|0 ; \mu, \nu\rangle \\
& -\left(a_{b}^{*}\right)^{-1} a_{b}|0 ; \mu, \nu\rangle=\frac{\nu}{\mu}|0 ; \mu, \nu\rangle .
\end{aligned}
$$

In section 5.2, we will derive these two equations again and reinterpret them from the viewpoint of the representation theory. In this framework, the operators $Q^{-1} P$ and $\left(a_{b}^{*}\right)^{-1} a_{b}$ have point spectra with continuous potency and they are constructed from an irreducible unitary representation of the algebra $\mathfrak{s u}(1,1)$ naturally. While the adjoints of these operators are not subnormal operators in the case of one-particle system, the adjoints of these operators are subnormal operators in the cases of two-particle system and multi-particle systems. Hence we can characterize a type of physically interpretable states by tensor-product, as the eigenvectors of the adjoints of subnormal operators in the cases of two-particle and multi-particle systems.

From a more general viewpoint, our investigation in this paper is regarded as a problem of the joint measurement between the self-adjoint part and the skewadjoint part of a subnormal operator which do not always commute with each other. However, we should be careful about the difference between self-adjoint operators and symmetric operators in these discussions, because there are many delicate problems when unbounded operators are treated (section 6.1).

In this paper, the complex conjugate and the adjoint operator are denoted by * And the closure is denoted by the overline.

\section{Subnormal operator and POVM}

In this section, we will summarize several well-known lemmas and will modify them for the discussion in the following sections. Some of the well-known lemmas will be extended for unbounded operators, and the proofs of the extended version will be given, as well. In this paper, only densely defined linear operator will be discussed. In the following, $\mathcal{D}_{o}(X)$ denotes the domain of a linear operator $X$. A densely defined operator $X$ is called closed if the domain $\mathcal{D}_{o}(X)$ is complete with respect to the graph norm $\|\phi\|_{\mathcal{D}_{o}(X)}:=\sqrt{\|\phi\|^{2}+\|X \phi\|^{2}}$. In operator theory, for two densely defined operator $X, Y$, the product $X Y$ is defined as $\phi \mapsto X(Y(\phi))$ for any vector $\phi$ belonging to the domain $\mathcal{D}_{o}(X Y):=\left\{\phi \in \mathcal{D}_{o}(X) \mid X \phi \in \mathcal{D}_{o}(Y)\right\}$. The notation $X \subset Y$ means that $\mathcal{D}_{o}(X) \subset \mathcal{D}_{o}(Y)$ and $X \phi=Y \phi, \phi \in \mathcal{D}_{o}(X)$. The notation $X=Y$, also means that $X \subset Y$ and $Y \subset X$. We will begin with reviewing the definition of normal operator and that of subnormal operator in unbounded case.

Definition 1 A closed operator $T$ on $\mathcal{H}$ is called normal if it satisfies the condition $T^{*} T=T T^{*}$. 
Remark that the operator $X^{*} X$ is defined on its domain $\mathcal{D}_{o}\left(X^{*} X\right):=\left\{\phi \in \mathcal{D}_{o}(X) \mid X \phi \in\right.$ $\left.\mathcal{D}_{o}\left(X^{*}\right)\right\}$ and it is self-adjoint and non-negative.

Definition 2 A closed operator $S$ is called subnormal if there exists a Hilbert space $\mathcal{K}$ including $\mathcal{H}$ and a normal operator $T$ on $\mathcal{K}$ such that $S=T P_{\mathcal{H}}$, where $P_{\mathcal{H}}$ denotes the projection from $\mathcal{K}$ to $\mathcal{H}$ and we write the operator $S P_{\mathcal{H}}$ on the bigger space $\mathcal{K}$ by $S$. In the following, we call the pair $(\mathcal{K}, T)$ a normal extension of the subnormal operator $S$.

Remark 1 Many papers, for example, Stochel and Szafraniec [6, 7], Szafraniec [8], Ôta [9] and Lahti, Pellonpää and Ylinen [10], adopt another definition of the subnormality, which substitutes $S \subset T P_{\mathcal{H}}$ for $S=T P_{\mathcal{H}}$. According to Ôta [9], there exists an example which is not subnormal in our definition, but subnormal in their definition.

For a spectral measure (i.e. a resolution of identity by projections) $E$ over $\mathbb{C}$, $\int_{\mathbb{C}} z E(d z)$ denotes the operator $\phi \mapsto \lim _{n \rightarrow \infty}\left(\int_{|z|<n} z E(d z) \phi\right)$ with the domain $\left\{\left.\phi \in \mathcal{H}\left|\int_{\mathbb{C}}\right| z\right|^{2}\langle\phi, E(d z) \phi\rangle<\infty\right\}$. Concerning normal operators, the following lemma is well-known. See Theorem 13.33 in Rudin [11.

Lemma 3 For a normal operator $T$, there uniquely exists a spectral measure $E_{T}$ over $\mathbb{C}$ such that $T=\int_{\mathbb{C}} z E_{T}(d z)$.

Lemma 3 tells that a normal operator corresponds to a spectral measure by one to one. Next, we will discuss measurements in a quantum system in order to investigate what is corresponding to Lemma 3 in the case of subnormal operators.

Let $\mathcal{H}$ be a Hilbert space representing a physical system of interest. Then, the state is denoted by a non-negative operator $\rho$ on $\mathcal{H}$ whose trace is 1 . It is called a density operator on $\mathcal{H}$, and the set of density operators on $\mathcal{H}$ is denoted by $\mathcal{S}(\mathcal{H})$. Let $P_{\rho}$ be the probability distribution given by a density $\rho$ and a measurement. Then, the probabilistic property of the measurement is described by the map $P: \rho \mapsto P_{\rho}$. We can naturally assume that the map $P$ satisfies the following condition from the formulation of quantum mechanics:

$$
\lambda P_{\rho_{1}}+(1-\lambda) P_{\rho_{2}}=P_{\lambda \rho_{1}+(1-\lambda) \rho_{2}}, \quad 0<\forall \lambda<1, \forall \rho_{1}, \rho_{2} \in \mathcal{S}(\mathcal{H})
$$

Lemma 4 For a map $P$ satisfying (3), there uniquely exists a positive operator valued measure (POVM) $M$ defined in the following which satisfies the condition

$$
P_{\rho}(B)=\operatorname{tr} M(B) \rho, \quad \forall B \in \mathcal{F}(\Omega), \forall \rho \in \mathcal{S}(\mathcal{H})
$$

This lemma was proved by Ozawa [12] in a more general framework. For an easy proof of a finite-dimensional case, see section 6 in chapter I of Holevo 13. This lemma guarantees that we have only to discuss POVMs in order to describe probabilistic properties.

Definition 5 Let $M$ be a map from a $\sigma$-field $\mathcal{F}(\Omega)$ over $\Omega$ to the set $\mathcal{B}_{s a}^{+}(\mathcal{H})$ of bounded, self-adjoint and non-negative operators on $\mathcal{H}$. The map $M$ is called a positive operator valued measure (POVM) on $\mathcal{H}$ over $\Omega$ if it satisfies the following:

- $M(\emptyset)=0, \quad M(\Omega)=I \quad(I$ : indentity op. $)$ 
Subnormal operators regarded as generalized observables

- $\sum_{i} M\left(B_{i}\right)=M\left(\cup_{i} B_{i}\right)$ for $B_{i} \cap B_{j}=\emptyset, \quad(i \neq j)$.

A POVM $M$ is a spectral measure if and only if $M(B)$ is a projection for any $B$. The following Lemma 6 is called Naímark's extension theorem. For a proof, see Naímark [14, section 5 in chapter II in Holevo [13] or Theorem 6.2.18 in Hiai and Yanagi [15]. It implies that the set of spectral measures is an important class in POVMs.

Lemma 6 Let $M$ be a POVM over a $\sigma$-field $\mathcal{F}(\Omega)$ on a Hilbert space $\mathcal{H}$. There exist a Hilbert space $\mathcal{K}$ including $\mathcal{H}$ and a spectral measure $E$ on the Hilbert space $\mathcal{K}$ such that

$$
M(B)=P_{\mathcal{H}} E(B) P_{\mathcal{H}}, \quad \forall B \in \mathcal{F}(\Omega),
$$

where $P_{\mathcal{H}}$ denotes the projection from $\mathcal{K}$ to $\mathcal{H}$. We call such a pair $(\mathcal{K}, E)$ a Naimark extension of the POVM $M$.

In the following, we will treat only POVMs over the complex numbers $\mathbb{C}$ whose $\sigma$-field is a family of Borel sets.

Definition 7 A closed subspace $\mathcal{H}^{\prime}$ of $\mathcal{H}$ is said to reduce a spectral measure $E$ on $\mathcal{H}$, if the projection $P_{\mathcal{H}^{\prime}}$ to $\mathcal{H}^{\prime}$ commutes with $E(B)$ for any Borel set $B$. A Naímark extension $(\mathcal{K}, E)$ of a POVM $M$ on $\mathcal{H}$ is called minimal if $\mathcal{K}$ has no non-trivial subspace which includes $\mathcal{H}$ and reduces the spectral measure $E$.

The following lemma guarantees the uniqueness of the minimal Naimark extension. It is proved as a corollary of PRINCIPAL THEOREM in section 6 of Appendix in Riesz and Sz.-Nagy 16.

Lemma 8 Let $\left(\mathcal{K}_{1}, E_{1}\right)$ and $\left(\mathcal{K}_{2}, E_{2}\right)$ be Naimark extensions of a POVM $M$ on $\mathcal{H}$. There exists a unitary map $V$ from $\mathcal{K}_{1}$ to $\mathcal{K}_{2}$ such that $U \phi=\phi$ for any $\phi \in \mathcal{H}$ and $V E_{1}(B) V^{*}=E_{2}(B)$ for any Borel $B$.

We will give the following definition with respect to the inequalities among linear operators not necessarily bounded.

Definition 9 For non-negative and self-adjoint operators $X, Y$ on $\mathcal{H}$, we denote $X \geq Y$ if they satisfy

$$
\langle\phi, X \phi\rangle \geq\langle\phi, Y \phi\rangle, \quad \forall \phi \in \mathcal{D}_{f}(q(X)) \subset \mathcal{D}_{f}(q(Y)) .
$$

where $q(X)$ denotes the closed non-negative quadratic form defined by a non-negative self-adjoint operator $X$ and $\mathcal{D}_{f}(q)$ denotes the domain of a closed non-negative quadratic form $q$.

We introduce the operators $\mathrm{E}(M)$ and $\mathrm{V}(M)$ on $\mathcal{H}$ which are represent formally $\int_{\mathbb{C}} z M(d z)$ and $\int_{\mathbb{C}}|z|^{2} M(d z)$, respectively. Later, by using Lemma 10, we will give more rigorous definition of $\mathrm{E}(M)$ and $\mathrm{V}(M)$. Then, for $\phi \in \mathcal{D}_{f}(\mathrm{q}(M)),\|\phi\|=1$ and a POVM $M$, the expectation of the measurement of the state by the POVM $M$ is $\langle\phi|\mathrm{E}(M)| \phi\rangle$ and the variance of it is $\langle\phi|\mathrm{V}(M)| \phi\rangle-|\langle\phi|\mathrm{E}(M)| \phi\rangle|^{2}$. It is sufficient to evaluate the operator $\mathrm{V}(M)$, in order to evaluate the variance. But, when they are 
unbounded, we should be more careful with respect to their domains. We define the closed non-negative quadratic form $\mathrm{q}(M)$ with the domain $\mathcal{D}_{f}(\mathrm{q}(M))$ by

$$
\begin{aligned}
& \mathrm{q}(M)(\phi, \phi):=\int_{\mathbb{C}}|z|^{2}\langle\phi, M(d z) \phi\rangle, \quad \phi \in \mathcal{D}_{f}(\mathrm{q}(M)) . \\
& \mathcal{D}_{f}(\mathrm{q}(M)):=\left\{\left.\phi \in \mathcal{H}\left|\int_{\mathbb{C}}\right| z\right|^{2}\langle\phi, M(d z) \phi\rangle<\infty\right\} .
\end{aligned}
$$

We assume the condition that the set $\mathcal{D}_{f}(\mathrm{q}(M))$ is a dense subset of $\mathcal{H}$. Let $\mathrm{V}(M)$ be the self-adjoint operator defined by the closed non-negative quadratic form $\mathrm{q}(M)$. Next, we will define the operator $\tilde{\mathrm{E}}(M)$. Define $\mathrm{E}_{R}(M):=\int_{|z|<R} z M(d z)$. Then, the sequence $\left\{\mathrm{E}_{n}(M) \phi\right\}$ is a Cauchy sequence for any $\phi \in \mathcal{D}_{f}(\mathrm{q}(M))$, because we have $\left\|\mathrm{E}_{n}(M) \phi-\mathrm{E}_{m}(M) \phi\right\|^{2}=\int_{n \leq|z|<m}|z|^{2}\langle\phi, M(d z) \phi\rangle$ for $n<m$. Therefore, we can define the vector $\tilde{\mathrm{E}}(M) \phi:=\lim _{n \rightarrow \infty} \mathrm{E}_{n}(M) \phi$. Thus, we can define the operator $\tilde{\mathrm{E}}(M)$ on the domain $\mathcal{D}_{f}(\mathrm{q}(M))$.

Lemma 10 The operator $\tilde{\mathrm{E}}(M)$ has a closed extension.

From this lemma, we can define the closed operator $\mathrm{E}(M)$ by the closure of the operator $\tilde{\mathrm{E}}(M)$.

Proof Let $(E, \mathcal{K})$ and $P_{\mathcal{H}}$ be a Naimark extension of $M$ and the projection from $\mathcal{K}$ to $\mathcal{H}$. The operator $T:=\int z E(d z)$ is normal. From the definition of $T$, we have $\mathcal{D}_{o}(T)=\left\{\left.\phi \in \mathcal{K}\left|\int\right| z\right|^{2}\langle\phi, E(d z) \phi\rangle<\infty\right\}$. Then the domain $\mathcal{D}_{f}(\mathrm{q}(M))$ equals $\mathcal{D}_{o}(T) \cap \mathcal{H}$. Let $T=U|T|$ be a polar decomposition of $T$. Since the operator $T$ is normal, we have $U|T|=|T| U$. This equation implies that the domain of $|T|$ is invariant under the action of $U$.

In general, for a closed operator $X$ on $\mathcal{K}$ and closed subset $\mathcal{H}$ of $\mathcal{K}$, the operator $X P_{\mathcal{H}}$ with the domain $\mathcal{D}_{o}(X) \cap \mathcal{H}$ is closed if $\mathcal{D}_{o}(X) \cap \mathcal{H}$ is dense in $\mathcal{H}$. We can define the closed operator $T^{*} P_{\mathcal{H}}$ on its domain $\mathcal{D}_{o}\left(T^{*} P_{\mathcal{H}}\right):=\mathcal{D}_{o}\left(T^{*}\right) \cap \mathcal{H}=\mathcal{D}_{o}(T) \cap \mathcal{H}=\mathcal{D}_{f}(\mathrm{q}(M))$. Then, we have the relation $\mathcal{D}_{o}\left(\left(T^{*} P_{\mathcal{H}}\right)^{*}\right) \supset \mathcal{D}_{o}(T)$. Define the closed operator $\left(T^{*} P_{\mathcal{H}}\right)^{*} P_{\mathcal{H}}$ on its domain $\mathcal{D}_{o}\left(\left(T^{*} P_{\mathcal{H}}\right)^{*} P_{\mathcal{H}}\right):=\mathcal{D}_{o}\left(\left(T^{*} P_{\mathcal{H}}\right)^{*}\right) \cap \mathcal{H} \supset \mathcal{D}_{o}(T) \cap \mathcal{H}=\mathcal{D}_{f}(\mathrm{q}(M))$. Then, we obtain $\left(T^{*} P_{\mathcal{H}}\right)^{*} P_{\mathcal{H}} \supset \tilde{\mathrm{E}}(M)$. It follows that the operator $\tilde{\mathrm{E}}(M)$ has a closed extension.

Lemma 11 Let $X$ and $M$ be an operator on a Hilbert space $\mathcal{H}$ and a POVM on the Hilbert space $\mathcal{H}$, respectively. If $X \supset \mathrm{E}(M)$, then we have $\mathrm{V}(M) \geq X^{*} X$.

Proof For a vector $\phi \in \mathcal{D}_{f}(\mathrm{q}(M))$, we have

$$
\mathrm{q}(M)(\phi, \phi)-\left\langle\phi\left|X^{*} X\right| \phi\right\rangle=\int_{\mathbb{C}}\left\langle\phi\left|\left(z^{*}-X^{*}\right) M(d z)(z-X)\right| \phi\right\rangle \geq 0 .
$$

Since the relation $\mathcal{D}_{f}(\mathrm{q}(M)) \subset \mathcal{D}_{o}(\mathrm{E}(M)) \subset \mathcal{D}_{o}(X)$ holds, we obtain Lemma 11 . The bounded version of this lemma is proved by Helstrom [17] from the viewpoint of quantum estimation theory. Its bounded version, also, follows from Kadison's inequality [18. 
Lemma 12 Let $S$ be an operator defined on the dense subset $\mathcal{D}_{o}(S)$ of $\mathcal{H}$. The operator $S$ is subnormal if and only if there exists a POVM $M$ satisfying the conditions

$$
\begin{aligned}
& S=\mathrm{E}(M) \\
& S^{*} S=\mathrm{V}(M) .
\end{aligned}
$$

Proof Let $(\mathcal{K}, T)$ and $P_{\mathcal{H}}$ be a normal extension of the operator $S$ and the projection from $\mathcal{K}$ to $\mathcal{H}$, respectively. By defining a POVM $M$ by $M(B):=P_{\mathcal{H}} E_{T}(B) P_{\mathcal{H}}$, the equation (4) is trivial. Since the equation $\mathrm{V}(M)=\left(T P_{\mathcal{H}}\right)^{*}\left(T P_{\mathcal{H}}\right)=S^{*} S$ holds, we have the equation (5). Assume the equations (4) and (5). From Naímark's extension theorem (Lemma 6) there exists a Naïmark extension $(\mathcal{K}, E)$ of the POVM $M$. Define a normal operator $T:=\int_{\mathbb{C}} z E(d z)$. Then we have $\mathrm{V}(M)=\left(T P_{\mathcal{H}}\right)^{*}\left(T P_{\mathcal{H}}\right), \mathrm{E}(M)=P_{\mathcal{H}}\left(T P_{\mathcal{H}}\right)$. From the equations (4), (5) and Lemma 14, we can prove that $S$ is subnormal.

The bounded version of this lemma is proved by Bram [19].

Definition 13 A POVM $M$ is called a $P O V M$ of a subnormal operator $S$ if $M$ satisfies the preceding conditions (4) and (5).

We will prove Lemma 14 applied in the proof of Lemma 12.

Lemma 14 Let $S, \mathcal{K}$ and $P_{\mathcal{H}}$ be an operator on a Hilbert space $\mathcal{H}$, a Hilbert space including the Hilbert space $\mathcal{H}$ and the projection from $\mathcal{K}$ to $\mathcal{H}$, respectively. For an operator $T$ on $\mathcal{K}$, the following are equivalent:

(A) $S=T P_{\mathcal{H}}$.

(B) $S^{*} S=\left(T P_{\mathcal{H}}\right)^{*}\left(T P_{\mathcal{H}}\right), \quad S=P_{\mathcal{H}}\left(T P_{\mathcal{H}}\right)$.

Proof It is easy to derive the condition (B) from the condition (A). Assume the condition $(\mathbf{B})$. We have $\left(T P_{\mathcal{H}}\right)^{*}\left(T P_{\mathcal{H}}\right)=\left(P_{\mathcal{H}}\left(T P_{\mathcal{H}}\right)\right)^{*}\left(P_{\mathcal{H}}\left(T P_{\mathcal{H}}\right)\right)+$ $\left(\left(I-P_{\mathcal{H}}\right) T P_{\mathcal{H}}\right)^{*}\left(\left(I-P_{\mathcal{H}}\right)\left(T P_{\mathcal{H}}\right)\right)$ and $\left(P_{\mathcal{H}}\left(T P_{\mathcal{H}}\right)\right)^{*}\left(P_{\mathcal{H}}\left(T P_{\mathcal{H}}\right)\right)=S^{*} S=\left(T P_{\mathcal{H}}\right)^{*}\left(T P_{\mathcal{H}}\right)$. Therefore, we obtain $\left(I-P_{\mathcal{H}}\right)\left(T P_{\mathcal{H}}\right)=0$. Thus, we get the condition $(\mathbf{A})$.

Definition 15 A closed subspace $\mathcal{H}^{\prime}$ of $\mathcal{H}$ is said to reduce a normal operator $T$ on $\mathcal{H}$, if the closed subspace $\mathcal{H}^{\prime}$ of $\mathcal{H}$ reduce its spectral measure $E_{T}$. This condition is equivalent to the condition that the projection $P_{\mathcal{H}^{\prime}}$ commutes with the operators $U, U^{*}$ and $e^{i t|T|^{2}}$ for any real number $t$, where $T=U|T|$ is the polar decomposition of $T$ with unitary $U$. A normal extension $(T, \mathcal{K})$ of a subnormal operator $S$ on $\mathcal{H}$ is called minimal if $\mathcal{K}$ has no non-trivial subspace which includes $\mathcal{H}$ and reduces the normal operator $T$.

The POVM $M(B):=P_{\mathcal{H}} E_{T}(B) P_{\mathcal{H}}$ can be defined for a normal extension $(T, \mathcal{K})$ of a subnormal operator $S$, and it is a POVM of $S$. Conversely, from Lemma 8 , if the normal extension $(T, \mathcal{K})$ is minimal, the spectral measure $E_{T}$ is unitarily equivalent with the minimal Naímark extension of $M$. Therefore, there exists a one-to-one correspondence between minimal normal extensions of a subnormal operator $S$ and its POVMs. 
Subnormal operators regarded as generalized observables

Lemma 16 A normal extension $(T, \mathcal{K})$ of a subnormal operator $S$ on $\mathcal{H}$ is minimal if and only if $\mathcal{K}=\overline{\mathcal{L}}$ where the subspaces $\mathcal{L}$ and $\mathcal{C}$ of $\mathcal{K}$ is defined as

$$
\begin{aligned}
& \mathcal{L}:=\left\{\sum_{k=1}^{n}\left(U^{*}\right)^{k} \psi_{k} \mid \psi_{k} \in \overline{\mathcal{C}}, n \in \mathbb{N}\right\} \\
& \mathcal{C}:=\left\{\sum_{k=1}^{n} e^{i t_{k}|T|^{2}} \psi_{k} \mid \psi_{k} \in \mathcal{H}, t_{k} \in \mathbb{R}, n \in \mathbb{N}\right\} .
\end{aligned}
$$

where $T=U|T|$ is the polar decomposition of $T$ with unitary $U$.

Proof Assume that a closed subspace $\mathcal{K}^{\prime}$ of $\mathcal{K}$ including $\mathcal{H}$ reduces the normal operator $T$. Then, for any $h \in \mathcal{H}$, any integer $m$ and any real number $t$, we have $e^{i t|T|^{2}} h \in \mathcal{K}^{\prime}$. Since the closed subspace $\mathcal{K}^{\prime}$ includes $\mathcal{C}$, the closed subspace $\mathcal{K}^{\prime}$ includes $\overline{\mathcal{C}}$. Similarly, we can show that the closed subspace $\mathcal{K}^{\prime}$ includes $\overline{\mathcal{L}}$ from this fact.

Next, we will prove that the closed subspace $\overline{\mathcal{C}}$ is invariant for $U$. It is sufficient to show that $U \phi \in \overline{\mathcal{C}}$ for any $\phi \in \mathcal{H}$. From the definition of $\mathcal{C}$, the closure $\overline{\mathcal{C}}$ reduces the operator $|T|^{2}$. Also, it reduces the operators $|T|$ and $|T|^{-1}$. Since $\mathcal{D}_{o}\left(|T|^{-1}\right) \subset \operatorname{Im} T$, $U \phi=|T|^{-1} S \phi \in \overline{\mathcal{C}}$ holds for any $\phi \in \mathcal{D}_{o}(S)$. We have $U \mathcal{H} \subset \overline{\mathcal{C}}$ because $U$ is bounded and $\mathcal{D}_{o}(S)$ is dense in $\mathcal{H}$. Thus, $U e^{i t|T|^{2}} \phi=e^{i t|T|^{2}} U \phi \in \underline{\mathcal{C}}$ for any $\phi \in \mathcal{H}$. It follows that $\mathcal{C}$ is invariant for $U$.

Therefore, we have the relations $U \overline{\mathcal{L}} \subset \overline{\mathcal{L}}, U^{*} \overline{\mathcal{L}} \subset \overline{\mathcal{L}}$ and $e^{i t|T|^{2}} \overline{\mathcal{L}} \subset \overline{\mathcal{L}}$ for any real number $t$. These imply that $\left[P_{\overline{\mathcal{L}}}, U\right]=0,\left[P_{\overline{\mathcal{L}}}, U^{*}\right]=0$ and $\left[P_{\overline{\mathcal{L}}}, e^{i t|T|^{2}}\right]=0$. It follows that the closed subspace $\overline{\mathcal{L}}$ reduces the normal operator $T$. The lemma is now immediate.

Lemma 17 Let $(T, \mathcal{K})$ be a minimal normal extension of a subnormal operator $S$ on $\mathcal{H}$. A Hilbert space $\mathcal{K}^{\prime}$ including $\mathcal{H}$ and a normal operator $T^{\prime}$ satisfy the condition $S \subset T^{\prime} P_{\mathcal{H}}$. The following three conditions are equivalent.

(A) $\left\langle\phi_{1}, e^{i t|T|^{2}} \phi_{2}\right\rangle=\left\langle\phi_{1}, e^{i t\left|T^{\prime}\right|^{2}} \phi_{2}\right\rangle$ holds for any $\phi_{1}, \phi_{2} \in \mathcal{H}$.

(B) $\left\langle\phi_{1}, e^{i t|T|} \phi_{2}\right\rangle=\left\langle\phi_{1}, e^{i t\left|T^{\prime}\right|} \phi_{2}\right\rangle$ holds for any $\phi_{1}, \phi_{2} \in \mathcal{H}$.

(C) There exists an isometric map $V$ from $\mathcal{K}$ to $\mathcal{K}^{\prime}$ such that $V \phi=\phi$ for any $\phi \in \mathcal{H}$ and $V T V^{*}=T^{\prime} P_{\operatorname{Im} V}$.

The condition $(\mathbf{C})$ implies that $T^{\prime} P_{\mathcal{H}}=S$ i.e. the pair $\left(T^{\prime}, \mathcal{K}^{\prime}\right)$ is a normal extension of $S$.

Proof It is easy to show that the conditions $(\mathbf{A})$ and $(\mathbf{B})$ follows from the condition (C). First, we prove that the condition (A) implies the condition (B). Define the subspace $\mathcal{C}^{\prime}$ of $\mathcal{K}^{\prime}$ by $\mathcal{C}^{\prime}:=\left\{\sum_{k=1}^{n} e^{i t_{k}\left|T^{\prime}\right|^{2}} \psi_{k} \mid \psi_{k} \in \mathcal{H}, t_{k} \in \mathbb{R}, n \in \mathbb{N}\right\}$.

Similarly to Proof of Lemma 16, we can prove that the closure $\overline{\mathcal{C}}$ reduces $|T|^{2}$ and the closure $\overline{\mathcal{C}^{\prime}}$ reduces $\left|T^{\prime}\right|^{2}$. Then, the closures $\overline{\mathcal{C}}$ and $\overline{\mathcal{C}^{\prime}}$ reduce the operators $|T|$ and $\left|T^{\prime}\right|$, respectively. From the condition $(\mathbf{A}),\left\langle e^{i t_{1}|T|^{2}} \phi_{1}, e^{i t_{2}|T|^{2}} \phi_{2}\right\rangle=\left\langle e^{i t_{1}|T|^{2}} \phi_{1}, e^{i t_{2}\left|T^{\prime}\right|^{2}} \phi_{2}\right\rangle$ 
holds for any $\phi_{1}, \phi_{2} \in \mathcal{H}$ and any real numbers $t_{1}, t_{2}$. Therefore, we can define the unitary map $V_{\mathcal{C}}$ from $\overline{\mathcal{C}}$ to $\overline{\mathcal{C}}^{\prime}$ by

$$
V_{\mathcal{C}}\left(\sum_{k=1}^{n} e^{i t_{k}|T|^{2}} \phi_{k}\right)=\sum_{k=1}^{n} e^{i t_{k}\left|T^{\prime}\right|^{2}} \phi_{k}
$$

Thus, we have $V_{\mathcal{C}}|T|^{2} V_{\mathcal{C}}^{*}=\left|T^{\prime}\right|^{2}$ on $\overline{\mathcal{C}^{\prime}}$. It implies that $V_{\mathcal{C}}|T| V_{\mathcal{C}}^{*}=\left|T^{\prime}\right|$ on $\overline{\mathcal{C}^{\prime}}$ because the closures $\overline{\mathcal{C}}$ and $\overline{\mathcal{C}^{\prime}}$ reduce the operators $|T|$ and $\left|T^{\prime}\right|$, respectively. Since $V_{\mathcal{C}} \phi=\phi$ for any $\phi \in \mathcal{H}$ and $V_{\mathcal{C}} e^{i t|T|} V_{\mathcal{C}}^{*}=e^{i t\left|T^{\prime}\right|}$ for any $t \in \mathbb{R}$, the condition $(\mathbf{B})$ holds. Similarly, we can prove that the condition $(\mathbf{B})$ implies the condition $(\mathbf{A})$.

Next, we prove that the condition $(\mathbf{A})$ implies the condition $(\mathbf{C})$. From the above discussion, we can define the inverses $|T|^{-1}$ and $\left|T^{\prime}\right|^{-1}$ on $\operatorname{Im}|T| \cap \overline{\mathcal{C}}$ and $\operatorname{Im}\left|T^{\prime}\right| \cap \overline{\mathcal{C}^{\prime}}$, respectively. Then we have $V_{\mathcal{C}}|T|^{-1} V_{\mathcal{C}}^{*}=\left|T^{\prime}\right|^{-1}$ on $\operatorname{Im}\left|T^{\prime}\right| \cap \overline{\mathcal{C}^{\prime}}$.

Let $T=U|T|$ and $T^{\prime}=U^{\prime}\left|T^{\prime}\right|$ be the polar decompositions of $T$ and $T^{\prime}$ satisfying that $U$ and $U^{\prime}$ are unitary, respectively. The image $\operatorname{Im}|T|$ is invariant under the unitary transformation $U$, and the image $\operatorname{Im}\left|T^{\prime}\right|$ is invariant under $U^{\prime}$. Then, we have $\operatorname{Im} S \subset \operatorname{Im}|T| \cap \mathcal{H}$ Similarly, we have $\operatorname{Im} S \subset \operatorname{Im}\left|T^{\prime}\right| \cap \mathcal{H}$. Thus, for any $\phi \in \operatorname{Im} S$, we have $V_{\mathcal{C}}|T|^{-1} \phi=\left|T^{\prime}\right|^{-1} \phi$. From the proof of Lemma 16, the closed subspaces $\overline{\mathcal{C}}$ and $\overline{\mathcal{C}}$, are invariant for $U$ and $U^{\prime}$, respectively. For any $\phi_{1}, \phi_{2} \in \mathcal{D}_{o}(S)$, we have

$$
\begin{aligned}
\left\langle e^{i t|T|^{2}} \phi_{1}, U \phi_{2}\right\rangle & =\left\langle V_{\mathcal{C}} e^{i t|T|^{2}} \phi_{1}, V_{\mathcal{C}} U \phi_{2}\right\rangle=\left\langle V_{\mathcal{C}} e^{i t|T|^{2}} \phi_{1}, V_{\mathcal{C}}|T|^{-1} S \phi_{2}\right\rangle \\
& =\left\langle V_{\mathcal{C}} e^{i t|T|^{2}} V_{\mathcal{C}}^{*} V_{\mathcal{C}} \phi_{1}, V_{\mathcal{C}}|T|^{-1} V_{\mathcal{C}}^{*} V_{\mathcal{C}} S \phi_{2}\right\rangle=\left\langle e^{i t\left|T^{\prime}\right|^{2}} V_{\mathcal{C}} \phi_{1},\left|T^{\prime}\right|^{-1} V_{\mathcal{C}} S \phi_{2}\right\rangle \\
& =\left\langle e^{i t\left|T^{\prime}\right|^{2}} \phi_{1},\left|T^{\prime}\right|^{-1} S \phi_{2}\right\rangle=\left\langle e^{i t\left|T^{\prime}\right|^{2}} \phi_{1}, U^{\prime} \phi_{2}\right\rangle .
\end{aligned}
$$

Since $e^{i t|T|^{2}}, e^{i t\left|T^{\prime}\right|^{2}}, U$ and $U^{\prime}$ are bounded,

$$
\left\langle e^{i t|T|^{2}} \phi_{1}, U \phi_{2}\right\rangle=\left\langle e^{i t\left|T^{\prime}\right|^{2}} \phi_{1}, U^{\prime} \phi_{2}\right\rangle
$$

holds, for any $\phi_{1}, \phi_{2} \in \mathcal{H}$. Also, we can prove

$$
\left\langle\left(\sum_{k=1}^{n} e^{i t_{k}|T|^{2}} \psi_{k}\right), U\left(\sum_{k=1}^{n} e^{i t_{k}^{\prime}|T|^{2}} \psi_{k}^{\prime}\right)\right\rangle=\left\langle\left(\sum_{k=1}^{n} e^{i t_{k}\left|T^{\prime}\right|^{2}} \psi_{k}\right), U^{\prime}\left(\sum_{k=1}^{n} e^{i t_{k}^{\prime}\left|T^{\prime}\right|^{2}} \psi_{k}^{\prime}\right)\right\rangle,
$$

for arbitrary $\psi_{k}, \psi_{k}^{\prime} \in \mathcal{H}, t_{k}, t_{k}^{\prime} \in \mathbb{R}$. Therefore,

$$
\left\langle\phi_{1}, U \phi_{2}\right\rangle=\left\langle V_{\mathcal{C}} \phi_{1}, U^{\prime} V_{\mathcal{C}} \phi_{2}\right\rangle
$$

holds for any $\phi_{1}, \phi_{2} \in \overline{\mathcal{C}}$. Since the closed subspace $\overline{\mathcal{C}}$ is invariant for $U$ and the operator $U$ is bounded,

$$
\left\langle\phi_{1}, U^{n} \phi_{2}\right\rangle=\left\langle V_{\mathcal{C}} \phi_{1}, U^{\prime n} V_{\mathcal{C}} \phi_{2}\right\rangle
$$

holds for any $\phi_{1}, \phi_{2} \in \overline{\mathcal{C}}$ and any $n \in \mathbb{N}$.

We can define the isometric map $V$ from $\mathcal{K}=\overline{\mathcal{L}}$ to $\mathcal{K}^{\prime}$ by

$$
V\left(\sum_{k=1}^{n}\left(U^{*}\right)^{k} \psi_{k}\right)=\left(\sum_{k=1}^{n}\left(U^{\prime *}\right)^{k} \psi_{k}\right)
$$

where $\psi_{k}$ is an arbitrary element of $\overline{\mathcal{C}}$. It can be confirmed that this definition is welldefined from (6). Now, we can easily check the condition (C). 
Definition 18 A vector $\phi \in \mathcal{D}^{\infty}(X):=\cap_{n=0}^{\infty} \mathcal{D}_{o}\left(X^{n}\right)$ is called an analytic vector of $X$ if

$$
\sum_{i=0}^{\infty} \frac{t^{i}}{i}\left\|X^{i} \phi\right\|<\infty
$$

for any $t \in \mathbb{R}$. The set of all analytic vectors of $S$ is written by $\mathcal{A}(S)$.

Lemma 19 Assume that the set $\mathcal{A}(S)$ is dense in $\mathcal{H}$ for a subnormal operator $S$ on $\mathcal{H}$. Let $(T, K)$ be a normal normal extension of $S$. If a Hilbert space $\mathcal{K}^{\prime}$ including $\mathcal{H}$ and a normal operator $T^{\prime}$ satisfy the condition $T^{\prime} P_{\mathcal{H}} \supset S$, there exists an isometric map $V$ from $\mathcal{K}$ to $\mathcal{K}^{\prime}$ such that $V \phi=\phi$ for any $\phi \in \mathcal{H}$ and $V T V^{*}=T^{\prime} P_{\operatorname{Im} V}$. This implies that $T^{\prime} P_{\mathcal{H}}=S$ i.e. the pair $\left(T^{\prime}, \mathcal{K}^{\prime}\right)$ is a normal extension of $S$. Therefore, this assumption guarantees the uniqueness of the minimal normal extension.

This lemma shows that under the assumption, the pair $(T, \mathcal{H})$ is a normal extension of $S$ if a normal operator $T$ on $\mathcal{K}$ including $\mathcal{H}$ satisfies $T P_{\mathcal{H}} \supset S$. For a simple proof in the bounded case, see section 2 in chapter II of Conway [20]. Szafraniec [8] shows the uniqueness of the minimal normal extension under another assumption that any vector $\phi \in \mathcal{D}(S)$ satisfies (7) for some a real number $t>0$. Stochel and Szafraniec[7] discuss different sufficiently conditions for the uniqueness of the minimal normal extension. Proof It is sufficient to show that the condition (A) in Lemma 17 holds. Schwarz's inequality guarantees

$$
\sum_{k=0}^{\infty} \frac{t^{k}}{k !}\left\langle S^{k} \phi_{1}, S^{k} \phi_{2}\right\rangle<\sqrt{\sum_{k=0}^{\infty} \frac{t^{k}}{k !}\left\|S^{k} \phi_{1}\right\|^{2}} \sqrt{\sum_{k=0}^{\infty} \frac{t^{k}}{k !}\left\|S^{k} \phi_{2}\right\|^{2}}<\infty .
$$

for any $\phi_{1}, \phi_{2} \in \mathcal{A}(S)$ and any real number $t$. From Fubini's Theorem,

$$
\begin{aligned}
\left\langle\phi_{1}, e^{i t|T|^{2}} \phi_{2}\right\rangle & =\left\langle\phi_{1}, \sum_{k=0}^{\infty} \frac{\left(i t|T|^{2}\right)^{k}}{k !} \phi_{2}\right\rangle=\left\langle\phi_{1}, \sum_{k=0}^{\infty} \frac{(i t)^{k}\left(T^{*}\right)^{k} T^{k}}{k !} \phi_{2}\right\rangle \\
& =\sum_{k=0}^{\infty} \frac{(i t)^{k}}{k !}\left\langle S^{k} \phi_{1}, S^{k} \phi_{2}\right\rangle=\left\langle\phi_{1}, e^{i t\left|T^{\prime}\right|^{2}} \phi_{2}\right\rangle .
\end{aligned}
$$

From (8) and the fact that the operators $e^{i t|T|^{2}}$ and $e^{i t\left|T^{\prime}\right|^{2}}$ are bounded and $\mathcal{A}(S)$ is dense in $\mathcal{H}$, we have the equation $P_{\mathcal{H}} e^{i t|T|^{2}} P_{\mathcal{H}}=P_{\mathcal{H}} e^{i t\left|T^{\prime}\right|^{2}} P_{\mathcal{H}}$. Therefore, the condition (A) in Lemma 19 holds.

From the one-to-one correspondence between POVMs of a subnormal operator $S$ and its minimal normal extensions, we have the following corollary.

Corollary 20 For any subnormal operator S satisfying the assumption of Lemma 19, there uniquely exists the POVM M satisfying the equations (4) and (5).

Subnormal operators have the following properties:

Lemma 21 Let $S$ be a subnormal operator on $\mathcal{H}$. Then

$$
S^{*} S \geq S S^{*}
$$


Proof We have $\left(P_{\mathcal{H}} T\right)\left(P_{\mathcal{H}} T\right)^{*} \geq\left(P_{\mathcal{H}}\left(P_{\mathcal{H}} T\right)\right)\left(P_{\mathcal{H}}\left(P_{\mathcal{H}} T\right)\right)^{*}=S S^{*}$. From the normality of $T$, we have $S^{*} S=\left(T P_{\mathcal{H}}\right)^{*}\left(T P_{\mathcal{H}}\right)=\left(T^{*} P_{\mathcal{H}}\right)^{*}\left(T^{*} P_{\mathcal{H}}\right)$. Since $T^{*} P_{\mathcal{H}} \subset\left(P_{\mathcal{H}} T\right)^{*}($ See Theorem 13.2 in Rudin 11$])$, the inequality $\left(T^{*} P_{\mathcal{H}}\right)^{*}\left(T^{*} P_{\mathcal{H}}\right) \geq\left(P_{\mathcal{H}} T\right)\left(P_{\mathcal{H}} T\right)^{*}$ holds. Therefore, $S^{*} S \geq\left(P_{\mathcal{H}} T\right)\left(P_{\mathcal{H}} T\right)^{*} \geq S S^{*}$.

Operators satisfying (9) are called hyponormal operators $\mathbb{\|}$ and the class of these operators is important in the operator theory. The following Lemma 22 shows a relation between the POVM of a subnormal operator and an over-complete eigenvector system.

Lemma 22 Let $J$ and $K$ be an operator on $\mathcal{H}$ and a subset of complex numbers $\mathbb{C}$, respectively. Assume that there exists a vector $|z\rangle \in \mathcal{D}_{o}(J)$ satisfying $J|z\rangle=z|z\rangle$ for any complex number $z \in K$, and there exists a measure $\mu$ on $K$ satisfying $\int_{K}\left|z^{*}\right\rangle\left\langle z^{*}\right| \mu(d z)=I$. Then, $J^{*}$ is subnormal and the POVM $\left|z^{*}\right\rangle\left\langle z^{*}\right| \mu(d z)$ is the POVM of the subnormal operator $J^{*}$.

Proof From the assumptions, we have

$$
J^{*}=\int_{K}\left|z^{*}\right\rangle\left\langle z^{*}\left|\mu(d z) J^{*}=\int_{K} z\right| z^{*}\right\rangle\left\langle z^{*}\right| \mu(d z) .
$$

Note that $\left\langle z^{*}\right| J^{*}=z\left\langle z^{*}\right|$. Thus, The POVM $M(d z):=\left|z^{*}\right\rangle\left\langle z^{*}\right| \mu(d z)$ satisfies the condition (4). Therefore, we obtain

$$
J J^{*}=\int_{K} J\left|z^{*}\right\rangle\left\langle\left. z^{*}\left|J^{*} \mu(d z)=\int_{K}\right| z\right|^{2} \mid z^{*}\right\rangle\left\langle z^{*}\right| \mu(d z)=\mathrm{V}(M) .
$$

Then the POVM $M$ satisfies the condition (5) and the operator $J^{*}$ is subnormal, as shown from Lemma 12. We can confirm that the POVM $\left|z^{*}\right\rangle\left\langle z^{*}\right| \mu(d z)$ is the POVM of the subnormal operator $J^{*}$.

In the following of this section, we treat a relation between a subnormal operator and its spectrum.

Lemma 23 Let $S$ and $\phi$ be a subnormal operator and an eigenvector of $S$, respectively. Then, a vector $\phi$ is an eigenvector of the adjoint $S^{*}$ operator of $S$.

Proof Let $(\mathcal{K}, T)$ and $P_{\mathcal{H}}$ be a normal extension of $S$ and the projection from $\mathcal{K}$ to $\mathcal{H}$, respectively. Assume that $\phi \in \mathcal{D}_{o}(S)$ is an eigenvector of $S$ associated with an eigenvalue $c$ such that $\|\phi\|=1$. Since the equation $T \phi=c \phi$ holds, we have $T^{*} \phi=c^{*} \phi$. Thus $S^{*}=P_{\mathcal{H}} T^{*}$. Therefore we get $S^{*} \phi=c^{*} \phi$. Now, we obtain the Lemma.

Definition 24 A subnormal operator $S$ is called pure subnormal if it satisfies the following condition; if a subspace $\mathcal{I}$ of $\mathcal{H}$ satisfies that $S P_{\mathcal{I}}$ is subnormal, then the subspace $\mathcal{I}$ is $\{0\}$ or $\mathcal{H}$

Lemma 25 Any pure subnormal operator $S$ has no point spectrum.

\| This class was introduced by Halmos [2]. 
Proof Let $(\mathcal{K}, T), P_{\mathcal{H}}$ and $\phi$ be defined in Proof of Lemma 23. Since we have $S^{*} \phi=c^{*} \phi$, the operator $|\phi\rangle\langle\phi|$ commutes with the pure subnormal operator $S$. The fact contradicts the definition of pure subnormal operators.

According to Conway [20], it is sufficient to assume the purity and hyponormality in Lemma 25.

\section{Compound-system-type normal extension}

Now, as an example of a subnormal operator and its normal extension, we will treat the boson creation operator $a_{b}^{*}$ and the heterodyne measurement in quantum optics. The pair $\left(L^{2}(\mathbb{R}) \otimes L^{2}(\mathbb{R}), a_{b}^{*} \otimes I+I \otimes a_{b}\right)$ is a normal extension of the subnormal operator $a_{b}^{*}$ under the isometric embedding $L^{2}(\mathbb{R}) \rightarrow L^{2}(\mathbb{R}) \otimes L^{2}(\mathbb{R})$ defined by $\psi \mapsto \psi \otimes|0 ; 1,0\rangle$, where $|0 ; 1,0\rangle$ denotes the boson vacuum vector. Here, $a_{b}^{*} \otimes I+I \otimes a_{b}$ is a normal operator, and we have $\left(a_{b}^{*} \otimes I+I \otimes a_{b}\right) \phi \otimes|0 ; 1,0\rangle=\left(a_{b}^{*} \phi\right) \otimes|0 ; 1,0\rangle$ for any $\phi \in L^{2}(\mathbb{R})$. By substituting $a_{b}$ for $J$ in Lemma 22 and and by letting $|\alpha ; 1,0\rangle$ be the boson coherent state, we can confirm that $|\alpha ; 1,0\rangle\langle\alpha ; 1,0| d^{2} \alpha$ is the POVM of the subnormal operator $a_{b}^{*}$. The set of rapidly decreasing $C^{\infty}$ functions is dense in $L^{2}(\mathbb{R})$ and any rapidly decreasing $C^{\infty}$ function is analytic of $a_{b}^{*}$. Therefore, $a_{b}^{*}$ s POVM is uniquely determined.

The heterodyne measurement is implemented by the measurement of $a_{b}^{*} \otimes I+I \otimes a_{b}$ (i.e. the simultaneous measurement between $Q \otimes I+I \otimes Q$ and $P \otimes I-I \otimes P$ which commute with each other) under the circumstance where the state of basic system is $|\phi\rangle\langle\phi|$ and the state of the ancillary system is controlled to be the vacuum states $|0 ; 1,0\rangle\langle 0 ; 1,0|$. In detail, see section 6 in chapter III in Holevo [13 or section 6 in chapter $\mathrm{V}$ in Helstrom [17]. We will generalize normal extensions of similar type to this, by the name of compound-system-type normal extensions, as follows;

Definition 26 Let $S$ be a subnormal operator defined on a dense linear subspace $\mathcal{D}_{o}(S)$ of $\mathcal{H}$ and let $\mathcal{H}^{\prime}, T$ and $\psi$ be a Hilbert space, a normal operator defined on a dense subspace $\mathcal{D}_{o}(T)$ of the Hilbert space $\mathcal{H} \otimes \mathcal{H}^{\prime}$ and an element of $\mathcal{H}^{\prime}$ whose norm is unity, respectively. We call the triple $\left(\mathcal{H}^{\prime}, T, \psi\right)$ a compound-system-type normal extension of the subnormal operator $S$ if it satisfies the condition

$$
\mathcal{D}_{o}(S) \otimes \psi \subset \mathcal{D}_{o}(T), \quad(S \phi) \otimes \psi=T(\phi \otimes \psi), \quad \text { for any } \phi \in \mathcal{D}_{o}(S) .
$$

Thus the definition of the compound-system-type normal extension describes not only the probability distribution but also a framework of the concrete implementation process, while the definition of the normal extension given in section 2 describes only the probability distribution. Therefore, a compound-system-type normal extension contains more informations than the corresponding POVM.

In the following of this section, we discuss compound-system-type normal extensions of isometric operators and symmetric operators, where we let $\{|\uparrow\rangle,|\downarrow\rangle\}$ be a CONS of $\mathbb{C}^{2}$.

Lemma 27 An isometric operator $U$ defined on $\mathcal{H}$ is subnormal. Define the operator $T:=U \otimes|\uparrow\rangle\left\langle\uparrow\left|+U^{*} \otimes\right| \downarrow\right\rangle\left\langle\downarrow\left|+P_{\operatorname{Im} U^{\perp}} \otimes\right| \uparrow\right\rangle\langle\downarrow|$, where $\operatorname{Im} U^{\perp}$ denotes the orthogonal 
Subnormal operators regarded as generalized observables

complementary space of $\operatorname{Im} U$. Then, the operator $T$ is unitary on $\mathcal{H} \otimes \mathbb{C}^{2}$ and the triple $\left(\mathbb{C}^{2}, T,|\uparrow\rangle\right)$ is a compound-system-type normal extension of $U$.

Proof From the definition, we have

$$
\begin{aligned}
& T^{*} T=\left(U^{*} \otimes|\uparrow\rangle\langle\uparrow|+U \otimes| \downarrow\rangle\left\langle\downarrow\left|+P_{\operatorname{Im} U^{\perp}} \otimes\right| \downarrow\right\rangle\langle\uparrow|\right) \\
&\left(U \otimes|\uparrow\rangle\left\langle\uparrow\left|+U^{*} \otimes\right| \downarrow\right\rangle\left\langle\downarrow\left|+P_{\operatorname{Im} U^{\perp}} \otimes\right| \uparrow\right\rangle\langle\downarrow|\right) I_{\mathcal{H}} \otimes|\uparrow\rangle\left\langle\uparrow\left|+P_{\operatorname{Im} U} \otimes\right| \downarrow\right\rangle\left\langle\downarrow\left|+P_{\operatorname{Im} U^{\perp}} \otimes\right| \downarrow\right\rangle\langle\downarrow|=I_{\mathcal{H}} \otimes I_{\mathbb{C}^{2} .} \\
& T T^{*}=\left(U \otimes|\uparrow\rangle\left\langle\uparrow\left|+U^{*} \otimes\right| \downarrow\right\rangle\left\langle\downarrow\left|+P_{\operatorname{Im} U^{\perp}} \otimes\right| \uparrow\right\rangle\langle\downarrow|\right) \\
&\left(U^{*} \otimes|\uparrow\rangle\langle\uparrow|+U \otimes| \downarrow\rangle\left\langle\downarrow\left|+P_{\operatorname{Im} U^{\perp}} \otimes\right| \downarrow\right\rangle\langle\uparrow|\right) \\
&=P_{\operatorname{Im} U} \otimes|\uparrow\rangle\left\langle\uparrow\left|+I_{\mathcal{H}} \otimes\right| \downarrow\right\rangle\left\langle\downarrow\left|+P_{\operatorname{Im} U^{\perp}} \otimes\right| \uparrow\right\rangle\langle\uparrow|=I_{\mathcal{H}} \otimes I_{\mathbb{C}^{2}} .
\end{aligned}
$$

Then, the operator $T$ is unitary. Moreover, we have $T(\phi \otimes|\uparrow\rangle)=(U \phi) \otimes|\uparrow\rangle$. Therefore, the triple $\left(\mathbb{C}^{2}, T,|\uparrow\rangle\right)$ is a compound-system-type normal extension of $U$.

A closed symmetric operator $X$ is called maximal symmetric, if there exists no symmetric operator $Y$ such that $X \varsubsetneqq Y$.

Lemma 28 A closed symmetric operator $X$ is subnormal on $\mathcal{H}$. Define the operator $T:=X^{*} \otimes|-\rangle\langle+|+X \otimes|+\rangle\langle-|$ on the domain $\mathcal{D}_{o}(T):=\mathcal{D}_{o}\left(X^{*}\right) \otimes|+\rangle \oplus \mathcal{D}_{o}(X) \otimes|-\rangle$ with $| \pm\rangle:=\frac{1}{\sqrt{2}}(|\uparrow\rangle \pm|\downarrow\rangle)$, for the maximal symmetric operator $X$ on $\mathcal{H}$. Then, $T$ is a self-adjoint operator and the triple $\left(\mathbb{C}^{2}, T,|\uparrow\rangle\right)$ is a compound-system-type normal extension of $X$.

The classification of (2nd) self-adjoint extensions of symmetric operators is given in section 5 in Naímark [21].

Proof We can confirm that $T$ is self-adjoint. $\mathcal{D}_{o}(T) \cap \mathcal{H} \otimes|\uparrow\rangle=\mathcal{D}_{o}(X) \otimes|\uparrow\rangle$ and $T(\phi \otimes|\uparrow\rangle)=(X \phi) \otimes(|-\rangle\langle+|+|+\rangle\langle-|)|\uparrow\rangle=(X \phi) \otimes|\uparrow\rangle=X \phi \otimes|\uparrow\rangle$ holds for any $\phi \in \mathcal{D}_{o}(X)$. The lemma is immediate.

For example, we apply the inequalities (9) in Lemma 21 to a symmetric operator. If $X$ is self-adjoint, we have $X^{*} X=X X^{*}$. But, if the operator $X$ have no self-adjoint extension, we have $X^{*} X \varsubsetneqq X X^{*}$. This fact isn’t contradictly to the inequalities (9).

We have the following lemma from the classification by Naimark and the following fact; any maximal symmetric operator is unitarily equivalent with $\left(I \otimes P^{+}\right) \oplus Y$ or $\left(I \otimes P^{-}\right) \oplus Y$, where $Y$ is a self-adjoint operator and $P^{+}$and $P^{-}$are the momentum operators on $L^{2}\left(\mathbb{R}^{+}\right)$and $L^{2}\left(\mathbb{R}^{-}\right)$, respectively. This fact follows from section 104 in Ahkiezer and Glazman 22].

Lemma 29 Any minimal normal (self-adjoint) extension of a closed symmetric operator $X$ is unitarily equivalent with each other if and only if $X$ is maximal symmetric.

Remark 2 Lemma 29 gives an example of subnormal operator such that its minimal normal extension is not unique in the sense of unitary equivalence. 
Subnormal operators regarded as generalized observables

\section{Irreducible unitary representations of the algebra $\mathfrak{s u}(1,1)$ and their coherent states}

In this section, from the minimal-weight-type unitary representations of the algebra $\mathfrak{s u}(1,1)$ (defined in this section), we will construct the corresponding subnormal operators canonically, and will investigate the relationship between the coherent states defined by Perelomov [4 and these subnormal operators.

Definition $30 \mathrm{~A}$ triplet $\left(E_{0}, E_{+}, E_{-}\right)$of skew-adjoint operators is called a unitary representation of the algebra $\mathfrak{s u}(1,1)$ if the relations

$$
\left[E_{0}, E_{ \pm}\right]= \pm 2 E_{ \pm}, \quad\left[E_{+}, E_{-}\right]=E_{0}
$$

hold.

For the reason for this definition, see Remark 3. However, it is difficult to discuss the unitary representation in this notation because three operators $E_{0}, E_{+}, E_{-}$have no eigenvector. Thus, we define another triplet $\left(L_{0}, L_{+}, L_{-}\right)$by

$$
L_{0}:=i\left(E_{-}-E_{+}\right), \quad L_{ \pm}:=\frac{1}{2}\left(E_{0} \pm i\left(E_{+}+E_{-}\right)\right) .
$$

Then, this triplet satisfies the commutation relations of the same type

$$
\left[L_{0}, L_{ \pm}\right]= \pm 2 L_{ \pm}, \quad\left[L_{+}, L_{-}\right]=L_{0} .
$$

For this triplet,

$$
L_{0}^{*}=L_{0}, \quad L_{+}^{*}=-L_{-}
$$

hold, where $L_{+}$and $L_{-}$are neither self-adjoint nor skew-adjoint. Conversely, from the triplet $\left(L_{0}, L_{+}, L_{-}\right)$satisfying the conditions (13) and (14), a unitary representation $\left(E_{0}, E_{+}, E_{-}\right)$of the algebra $\mathfrak{s u}(1,1)$ can be constructed by

$$
E_{0}=L_{+}+L_{-}, \quad E_{ \pm}= \pm \frac{i}{2}\left(L_{0} \mp L_{+} \pm L_{-}\right) .
$$

The Casimir operator is useful for the analysis of the representation. In case of the algebra $\mathfrak{s u}(1,1)$, it is given by

$$
C:=E_{0}^{2}+2\left(E_{+} E_{-}+E_{-} E_{+}\right)=L_{0}^{2}+2\left(L_{+} L_{-}+L_{-} L_{+}\right) .
$$

For the general definition, see p.130-131 of Perelomov [4] or p.45 of Howe and Tan [23]. The relation (16) can be written in another form

$$
C=L_{0}^{2}-2 L_{0}+4 L_{+} L_{-}
$$

by using (13). From (11) and (13), the Casimir operator $C$ is commutative with $E_{0}, E_{+}, E_{-}, L_{0}, L_{+}$and $L_{-}$. From the Schur's lemma, in any irreducible representation, the Casimir operator $C$ is constant. 
Subnormal operators regarded as generalized observables

Lemma 31 Non-trivial irreducible unitary representations of $\mathfrak{s u}(1,1)$ are classified into the following three cases:

$$
\begin{array}{ll}
\text { Case } 1: & \operatorname{dim} L_{+}=0 \text { and } \operatorname{dim} L_{-}=1 \\
\text { Case } 2: & \operatorname{dim} L_{+}=1 \text { and } \operatorname{dim} L_{-}=0 \\
\text { Case } 3: & \operatorname{dim} L_{+}=0 \text { and } \operatorname{dim} L_{-}=0 .
\end{array}
$$

Case 2 is reduced to Case 1 , by exchanging $L_{-}$for $L_{+}$and by changing the sign of $L_{0}$. We will not treat Case 3 in this paper. Thus, only Case 1 will be discussed.

Proof The irreducibility requires that the dimensions of the kernels of $L_{-}$and $L_{+}$ are not more than one. Moreover, if the dimensions of both kernels are one, then the representation should be finite-dimensional. However, this circumstance is forbidden by the unitarity of the representation. Now, the lemma follows immediately.

Lemma 32 The unit vector $|0\rangle_{N}$ belonging to the Kernel of $L_{-}$is a eigen vector of $L_{0}$. this eigen value $\lambda$ is called the lowest weight and specifies the irreducible unitary representation of $\mathfrak{s u}(1,1)$ uniquely and satisfies $\lambda>0$. The equations

$$
\begin{aligned}
L_{0}|n\rangle_{N} & =(\lambda+2 n)|n\rangle_{N} \\
L_{+}|n\rangle_{N} & =\sqrt{(n+1)(\lambda+n)}|n+1\rangle_{N} \\
L_{-}|n\rangle_{N} & =-\sqrt{n(\lambda+n-1)}|n-1\rangle_{N}
\end{aligned}
$$

hold, where we define $|n\rangle_{N}:=\frac{1}{\|\left(L_{+}\right)^{n}|0\rangle_{N} \|}\left(L_{+}\right)^{n}|0\rangle_{N}$.

Proof Because the Casimir operator should be scalar-valued, we can show that $|0\rangle_{N}$ is the eigenvector of $L_{0}$, from (17).

Let $v_{n}:=\left(L_{+}\right)^{n}|0\rangle_{N}$. The commutation relations (13) yields the following relations;

$$
\begin{aligned}
& L_{0} v_{n}=(\lambda+2 n) v_{n} \\
& L_{+} v_{n}=v_{n+1} \\
& L_{-} v_{n}=-n(\lambda+n-1) v_{n-1}
\end{aligned}
$$

whence we can confirm that the lowest weight $\lambda$, with which $|0\rangle_{N}$ is associated, specifies the representation uniquely. From the above assumptions, we can confirm that the basis $\left\{v_{n}\right\}_{n=1}^{\infty}$ is complete and orthogonal. From the above relations, the Casimir operator $C$ is calculated to be the scalar $\lambda(\lambda-2)$. From the commutation relations (13), we have

$$
\left\langle v_{n}, v_{n}\right\rangle=n(\lambda+n-1)\left\langle v_{n-1}, v_{n-1}\right\rangle .
$$

Therefore, the equation

$$
|n\rangle_{N}=\sqrt{\frac{\Gamma(\lambda)}{n ! \Gamma(\lambda+n)}} v_{n}
$$

holds. Thus, (18) follows immediately. The unitarity of the representation guarantees $\lambda>0$. (See Theorem 1.1.5 in pp. 96 of Howe and Tan [23].)

In the following discussions, $\mathcal{H}_{\lambda}$ denotes the representation space of the irreducible unitary representation of $\mathfrak{s u}(1,1)$ characterized by the lowest weight $\lambda$. We call such a representation (i.e. Case 1) lowest-weight-type. The representation of the Lie group 
$\mathrm{SU}(1,1)$ can no more be constructed unless $\lambda$ is an integer than the representation of the Lie group $\mathrm{SO}(3)$ unless the total momentum is integer. (For more detail, see Remark 3 .) Especially, when the lowest weight $\lambda$ is an integer, the representation of the Lie group $\mathrm{SU}(1,1)$ is well-known as the discrete series [23, 24].

Definition 33 The operator $N:=\frac{1}{2}\left(L_{0}-\lambda\right)$ called $\mathfrak{s u}(1,1)$-number operator because of (18). The bounded operator $a:=\frac{1}{2} L_{+}^{-1}\left(L_{0}-\lambda\right)=L_{+}^{-1} N$ is called the $\mathfrak{s u}(1,1)$ annihilation operator. Its definition is well-defined because the vector $N|n\rangle_{N}$ belongs to the range of $L_{+}$for any $n$ and the kernel of $L_{+}$is $\{0\}$. The $\mathfrak{s u}(1,1)$-creation operator is defined by the adjoint $a^{*}$ of $a$.

The equations

$$
\begin{aligned}
a|n\rangle_{N} & =\sqrt{\frac{n}{n+\lambda-1}}|n-1\rangle_{N}, \\
a^{*}|n\rangle_{N} & =\sqrt{\frac{n+1}{n+\lambda}}|n+1\rangle_{N}
\end{aligned}
$$

hold, where we mean that $a|0\rangle_{N}=0$ by (19) in the exceptional case where $\lambda=1, n=0$, as a convention. From (19), the commutation relation $[a, N]=a$ is derived. From (19) and (20), we have

$$
\begin{aligned}
& a^{*} a=(N+\lambda-1)^{-1} N, \quad a a^{*}=(N+\lambda)^{-1}(N+1), \\
& {\left[a, a^{*}\right]=(\lambda-1)(N+\lambda)^{-1}(N+\lambda-1)^{-1}}
\end{aligned}
$$

for $\lambda \neq 1$, and

$$
a a^{*}=I, \quad a^{*} a=I-|0\rangle_{N}{ }_{N}\left\langle 0\left|, \quad\left[a, a^{*}\right]=\right| 0\right\rangle_{N}{ }_{N}\langle 0|
$$

instead of (21) for $\lambda=1$. Next, we will construct the $\mathfrak{s u}(1,1)$-coherent state as follows;

Definition 34 Introduce the unitary operator $U(\xi):=\exp \left(\xi L_{+}-\xi^{*} L_{+}^{*}\right)$ for a complex number $\xi$, according to Perelomov [4]. For the complex number $\zeta$ such that $|\zeta|<1$, we define the $\mathfrak{s u}(1,1)$-coherent state $|\zeta\rangle_{a}$ of the algebra $\mathfrak{s u}(1,1)$ by

$$
|\zeta\rangle_{a}:=U\left(\frac{1}{2} e^{i \arg \zeta} \ln \frac{1+|\zeta|}{1-|\zeta|}\right)|0\rangle_{N} .
$$

Squeezed states are characterized as $\mathfrak{s u}(1,1)$-coherent states, as we discuss in section 5.2 .

Lemma 35 The $\mathfrak{s u}(1,1)$-coherent state $|\zeta\rangle_{a}$ is an eigenvector of $a$, i.e. the equation

$$
a|\zeta\rangle_{a}=\zeta|\zeta\rangle_{a}
$$

holds.

Proof From the definition, we have

$$
\begin{aligned}
|\zeta\rangle_{a} & =\exp \left(\zeta L_{+}\right) \exp \left(\frac{1}{2} \ln \left(1-|\zeta|^{2}\right) L_{0}\right) \exp \left(\zeta^{*} L_{-}\right)|0\rangle_{N} \\
& =\left(1-|\zeta|^{2}\right)^{\lambda / 2} \exp \left(\zeta L_{+}\right)|0\rangle_{N},
\end{aligned}
$$


where see pp.73-74 of Perelomov [4] for the derivation of the first equation. Because we can show that $\left[a, L_{+}\right]=I$, we obtain the commutation relation $\left[a, \exp \left(\zeta L_{+}\right)\right]=$ $\zeta \exp \left(\zeta L_{+}\right)$. Moreover, from the relation $\exp \left(\frac{1}{2} \ln \left(1-|\zeta|^{2}\right) L_{0}\right) \exp \left(\zeta^{*} L_{-}\right)|0\rangle_{N}=$ $\left(1-|\zeta|^{2}\right)^{\lambda / 2}|0\rangle_{N}$, we have

$$
a|\zeta\rangle_{a}=\exp \left(\zeta L_{+}\right) a|0\rangle_{N}+\zeta \exp \left(\zeta L_{+}\right)|0\rangle_{N}=\zeta|\zeta\rangle_{a}
$$

Therefore, the coherent states of the algebra $\mathfrak{s u}(1,1)$ are characterized as the eigenvectors of the $\mathfrak{s u}(1,1)$-annihilation operator $a$.

Lemma 36 When $1>\lambda>0$, a and a are not subnormal. When $\lambda \geq 1$, $a^{*}$ is subnormal and $a$ is not subnormal. In this case, $a^{*}$ 's POVM is given by $(\lambda-1)\left|\zeta^{*}\right\rangle_{a}{ }_{a}\left\langle\zeta^{*}\right| \mu(d \zeta)$, where we define $\mu(d \zeta):=\frac{d^{2} \zeta}{\pi\left(1-|\zeta|^{2}\right)^{2}}$.

Proof When $\lambda>0$, it is shown that $a$ is not subnormal, from Lemma 23 and the fact that it has eigenvectors. When $\lambda<1$, it is shown that $a^{*}$ is not subnormal, from Lemma 21 and the fact that $\left[a, a^{*}\right] \geq 0$ does not hold. (See (21).)

Moreover, when $\lambda>1$, we can construct the resolution of the identity by the system of the coherent states:

$$
(\lambda-1) \int_{D}|\zeta\rangle_{a} a\langle\zeta| \mu(d \zeta)=I
$$

where $D$ denotes the unit disk $\{z \in \mathbb{C}|| z \mid<1\}$. From this resolution of the identity and Lemma 22, when $\lambda>1$, we can show that $a^{*}$ is a subnormal operator. On the other hand, when $\lambda \leq 1$, the integral in (26) diverges. However, the equations (22) imply that $a^{*}$ is isometric when $\lambda=1$. Then, $a^{*}$ is subnormal even when $\lambda=1$.

Definition 37 We formally define the operator

$$
A:=-i(a+1)(a-1)^{-1} .
$$

Since this operator is unbounded, we need more attention in this definition. First, define the unbounded operator $\tilde{A}$ by a linear fractional transform (Möbius transform) of $a$, as $\tilde{A}:=-i(a+1)(a-1)^{-1}$, where the domain $\mathcal{D}_{o}(\tilde{A})$ of $\tilde{A}$ is defined by $\left\langle\left\{|n\rangle_{N}\right\}_{n=0}^{\infty}\right\rangle$. The domain of $\tilde{A}^{*}$ is dense in $\mathcal{H}_{\lambda}$, as will be shown in the last part of Remark 6. Therefore, $\tilde{A}$ is closable and we can define the operator $A$ by $A:=\bar{A}=\tilde{A}^{* *}$. (See Reed and Simon [25.)

It is shown that $|\zeta\rangle_{a} \in \mathcal{D}_{o}(A)$ in the last part of Remark 6. Hence we have $A|\zeta\rangle_{a}=-i \frac{\zeta+1}{\zeta-1}|\zeta\rangle_{a}$. By defining $|\eta\rangle_{A}:=\left|\frac{\eta-i}{\eta+i}\right\rangle_{a}$, we can show that

$$
A|\eta\rangle_{A}=\eta|\eta\rangle_{A}
$$

holds. (Formally, the operator $a$ is the Cayley transform of $A$, with an appropriate discussion on its domain.)

Lemma 38 We have another expression of $A$ :

$$
A=\frac{1}{2} E_{+}^{-1}\left(E_{0}-\lambda\right) \text {. }
$$


Subnormal operators regarded as generalized observables

Proof From the relations $\left[a, L_{+}\right]=I$, (13), (15) and the definition of $a$, we can show that the relations

$$
\begin{aligned}
2\left(E_{0}-\lambda\right)(a-1) L_{+} & =\left(L_{+}+L_{-}-\lambda\right)\left(L_{0}-(\lambda-2)-2 L_{+}\right) \\
& =\left(L_{0}-L_{+}+L_{-}\right)\left(-\lambda+L_{0}+2+2 L_{+}\right) \\
& =-4 i E_{+}(a+1) L_{+} \\
\left(E_{0}-\lambda\right)(a-1)|0\rangle_{N} & =-\left(E_{0}-\lambda\right)|0\rangle_{N}=\left(L_{0}-L_{+}\right)|0\rangle_{N} \\
& =-2 i E_{+}|0\rangle_{N}=-2 i E_{+}(a+1)|0\rangle_{N}
\end{aligned}
$$

hold on $\mathcal{D}_{o}(\tilde{A})$. Hence, on $\mathcal{D}_{o}(\tilde{A})$, we have

$$
\left(E_{0}-\lambda\right)(a-1)=-2 i E_{+}(a+1) .
$$

By using (29), we obtain (28).

From (28), we have

$$
\left[A, A^{*}\right]=-(\lambda-1) E_{+}^{-2}
$$

formally, and

$$
A^{*} A-A A^{*}=(\lambda-1)\left(E_{+}^{-1}\right)^{*} E_{+}^{-1} \begin{cases}\text { on } \mathcal{D}_{o}\left(A A^{*}\right) & \text { for } \lambda \geq 1 \\ \text { on } \mathcal{D}_{o}\left(A^{*} A\right) & \text { for } 0<\lambda<1\end{cases}
$$

in more precise form. (The proof of this relation will be given in Remark 6.)

Lemma 39 When $1>\lambda>0, A$ and $A^{*}$ are not subnormal. When $\lambda \geq 1$, $A^{*}$ is subnormal and $A$ is not subnormal. In this case, $A^{*}$ 's POVM is given by $(\lambda-1)\left|\eta^{*}\right\rangle_{A}{ }_{A}\left\langle\eta^{*}\right| \nu(d \eta)$, where we define $\nu(d \eta):=\frac{d^{2} \eta}{4 \pi(\operatorname{Im} \eta)^{2}}$.

Proof For $\lambda>0$, from Lemma 23 and the fact that the operator $A$ has eigenvectors, it is shown that $A$ is not subnormal. When $\lambda<1, A^{*}$ is not subnormal because the relation (21) shows that the condition $A A^{*} \geq A^{*} A$ is not satisfied. Moreover, in a similar manner to the above discussion, the resolution of the identity by the eigenvectors of $A$

$$
(\lambda-1) \int_{\mathrm{H}}|\eta\rangle_{A}{ }_{A}\langle\eta| \nu(d \eta)=I
$$

holds when $\lambda>1$. Hence, when $\lambda>1$, we can show that $A^{*}$ is subnormal from Lemma 22. When $\lambda \leq 1$, the integral in (26) diverges. However, as will be proved in the last part of Remark 6, the operator $A^{*}$ is maximal symmetric when $\lambda=1$. From Lemma 28, $A^{*}$ is subnormal even when $\lambda=1$.

Remark 3 [Relation to unitary representations of $\mathrm{SU}(1,1)$ ] In the following, we discuss Definition 30 from the viewpoint of a unitary representation of the group $\mathrm{SU}(1,1)$. Any element $g$ in the group $\mathrm{SU}(1,1)$ is specified by two complex numbers $\nu(g)=$ $\nu_{1}(g)+\nu_{2}(g) i, \mu(g)=\mu_{1}(g)+\mu_{2}(g) i$ satisfying $|\nu(g)|^{2}-|\mu(g)|^{2}=1$ as

$$
g=\left(\begin{array}{ll}
\mu^{*}(g) & \nu(g) \\
\nu^{*}(g) & \mu(g)
\end{array}\right)=\left(\begin{array}{cc}
\mu_{1}(g)-\mu_{2}(g) i & \nu_{1}(g)+\nu_{2}(g) i \\
\nu_{1}(g)-\nu_{2}(g) i & \mu_{1}(g)+\mu_{2}(g) i
\end{array}\right) .
$$


The group $\mathrm{SU}(1,1)$ is isomorphic to the group $\mathrm{SL}(2, \mathbb{R})$ by the map

$$
j:\left(\begin{array}{cc}
\mu_{1}(g)-\mu_{2}(g) i & \nu_{1}(g)+\nu_{2}(g) i \\
\nu_{1}(g)-\nu_{2}(g) i & \mu_{1}(g)+\mu_{2}(g) i
\end{array}\right) \mapsto\left(\begin{array}{cc}
\mu_{1}(g)+\nu_{1}(g) & -\mu_{2}(g)-\nu_{2}(g) \\
\mu_{2}(g)-\nu_{2}(g) & \mu_{1}(g)-\nu_{1}(g)
\end{array}\right) .
$$

The (Lie) algebra $\mathfrak{s u}(1,1)$ associated with $\mathrm{SU}(1,1)$ is written by

$\mathfrak{s u}(1,1)=\left\{\left(\begin{array}{ll}a_{1,1} & a_{1,2} \\ a_{2,1} & a_{2,2}\end{array}\right) \mid\left(\begin{array}{cc}a_{1,1}^{*} & -a_{2,1}^{*} \\ -a_{1,2}^{*} & a_{2,2}^{*}\end{array}\right)=-\left(\begin{array}{cc}a_{1,1} & a_{1,2} \\ a_{2,1} & a_{2,2}\end{array}\right), a_{1,1}+a_{2,2}=0\right\}$.

The vector space $\mathfrak{s u}(1,1)$ has the following basis $e_{0}, e_{+}, e_{-}$as

$$
e_{0}=\left(\begin{array}{ll}
0 & 1 \\
1 & 0
\end{array}\right), e_{+}=\frac{i}{2}\left(\begin{array}{ll}
1 & -1 \\
1 & -1
\end{array}\right), e_{-}=\frac{i}{2}\left(\begin{array}{cc}
-1 & -1 \\
1 & 1
\end{array}\right) .
$$

Thus, from the isomorphism (31), we can naturally define the isomorphism $j_{*}$ from the algebra $\mathfrak{s u}(1,1)$ to the algebra $\mathfrak{s l}(2, \mathbb{R})$. Then, the image $j_{*}\left(e_{0}\right), j_{*}\left(e_{-}\right), j_{*}\left(e_{+}\right)$of the basis is written as

$$
j_{*}\left(e_{0}\right)=\left(\begin{array}{cc}
1 & 0 \\
0 & -1
\end{array}\right), j_{*}\left(e_{+}\right)=\left(\begin{array}{ll}
0 & 1 \\
0 & 0
\end{array}\right), j_{*}\left(e_{-}\right)=\left(\begin{array}{ll}
0 & 0 \\
1 & 0
\end{array}\right) .
$$

The basis $e_{0}, e_{-}, e_{+}$satisfies the following commutation relation:

$$
\left[e_{0}, e_{ \pm}\right]= \pm 2 e_{ \pm}, \quad\left[e_{+}, e_{-}\right]=e_{0}
$$

A map $V$ from a group $G$ to the set of unitary operators on a Hilbert space $\mathcal{H}$ is called a unitary representation of the group $G$ on $\mathcal{H}$ if

$$
V\left(g_{1} g_{2}\right)=V\left(g_{1}\right) V\left(g_{2}\right), \quad \forall g_{1}, g_{2} \in G .
$$

Let $\mathfrak{g}$ be the Lie algebra associated with a Lie group $G$. From a unitary representation of the group $G$ on a Hilbert space $\mathcal{H}$, we can naturally define the map $V_{*}$ from the Lie algebra $\mathfrak{g}$ to the set of skew-adjoint operators on $\mathcal{H}$, by

$$
V_{*}(X):=\left.\frac{d V(\exp (t X))}{d t}\right|_{t=0} .
$$

It satisfies that $V_{*}([X, Y])=\left[V_{*}(X), V_{*}(Y)\right]$. Then, a linear map $f$ from a Lie algebra $\mathfrak{g}$ to the the set of skew-adjoint operators on a Hilbert space $\mathcal{H}$ is called a unitary representation of the Lie algebra $\mathfrak{g}$ on $\mathcal{H}$ if

$$
[f(X), f(Y)]=f([X, Y]), \quad \forall X, Y \in \mathfrak{g} .
$$

We can construct the unitary representation $V$ of the universal covering group $\mathbb{G}$ associated with a Lie algebra $\mathfrak{g}$ from a unitary representation $f$ of $\mathfrak{g}$, by

$$
V(\exp X):=\exp f(X), \quad \forall X \in \mathfrak{g} .
$$

Since any element of the (Lie) algebra $\mathfrak{s u}(1,1)$ is described by a linear sum of bases $e_{0}, e_{+}, e_{-}$, we can uniquely construct the unitary representation of the algebra $\mathfrak{s u}(1,1)$ from a triplet $\left(E_{0}, E_{+}, E_{-}\right)$of skew-adjoint operators satisfying (11). Thus, we can regard the triplet $\left(E_{0}, E_{+}, E_{-}\right)$satisfying (11) as the unitary representation of the algebra $\mathfrak{s u}(1,1)$.

ๆ A group is called a universal covering group if it is connected and if its homotopy group is trivial. 
Remark 4 [Spectrums of $a, a^{*}, A, A^{*}$ ] The point spectrum $\sigma_{p}(a)$ is the open unit disk $D$ the continuous spectrum $\sigma_{c}(a)$ is the unit circle $S:=\{z \in \mathbb{C}|| z \mid=1\}$ and the residual spectra is the empty set. Moreover, from Lemma 45, the point spectrum $\sigma_{p}\left(a^{*}\right)$, the continuous spectrum $\sigma_{c}\left(a^{*}\right)$ and the residual spectrum $\sigma_{r}\left(a^{*}\right)$ of $a^{*}$ are the empty set, $S$ and $D$, respectively.

It is shown that the point spectrum $\sigma_{p}(A)$ of $A$ is the upper-half plane $H$, the continuous spectrum $\sigma_{c}(A)$ is the real axis $\mathbb{R}$ and the residual spectrum $\sigma_{r}(A)$ is the empty set. On the other hand, from Lemma 45, the point spectrum $\sigma_{p}\left(A^{*}\right)$, the continuous spectrum $\sigma_{c}\left(A^{*}\right)$ and the residual spectrum $\sigma_{r}\left(A^{*}\right)$ of $A^{*}$ are the empty set, $\mathbb{R}$ and $H$, respectively.

Remark 5 [Action of the group to operators $a, A$ ] First, we discuss the action to a operator $a$. We let $\pi_{\mathrm{SU}(1,1)}$ be the projection from the universal covering group $\widehat{\mathrm{SU}(1,1)}$ to the group $\mathrm{SU}(1,1)$, and let $U$ be the maximal Cartan subgroup of $\widehat{\mathrm{SU}(1,1)}$ i.e. the 1-parameter subgroup generated by $i L_{0}$.

The homogeneous space $\widehat{\mathrm{SU}(1,1)} / U$ is isomorphic to the open unit disk $D$ in the sense that an element $g$ of the group $\widehat{\mathrm{SU}(1,1)}$ acts on the open unit disk $D$ as

$$
\zeta \mapsto \frac{\mu^{*} \zeta+\nu}{\nu^{*} \zeta+\mu}, \quad \zeta \in D
$$

where we simply use the notations $\mu$ and $\nu$ instead of the complex numbers $\mu \circ \pi_{\mathrm{SU}(1,1)}(g)$ and $\nu \circ \pi_{\mathrm{SU}(1,1)}(g)$ with the functions $\mu$ and $\nu$ defined at the beginning of Remark 3 , respectively. We let $V$ be the representation of the group $\widehat{\mathrm{SU}(1,1)}$, defined by this representation of $\mathfrak{s u}(1,1)$. Then, we have

$V(g)|\zeta\rangle_{a}{ }_{a}\left\langle\zeta\left|V(g)^{*}=\right| \frac{\mu^{*} \zeta+\nu}{\nu^{*} \zeta+\mu}\right\rangle_{a}{ }_{a}\left\langle\frac{\mu^{*} \zeta+\nu}{\nu^{*} \zeta+\mu}\right|, \quad g \in \widehat{\mathrm{SU}(1,1)}, \zeta \in \mathbb{C}$.

Thus, for any element $g \in \widehat{\mathrm{SU}(1,1)}$ and any complex number $\zeta$, there exists a real number $\theta(g, \zeta)$ such that

$$
V(g)|\zeta\rangle_{a}=e^{i \theta(g, \zeta)}\left|\frac{\mu^{*} \zeta+\nu}{\nu^{*} \zeta+\mu}\right\rangle_{a} .
$$

the equations (24) and (35) implies that

$$
V(g)^{*} a V(g)|\zeta\rangle_{a}=\frac{\mu^{*} \zeta+\nu}{\nu^{*} \zeta+\mu}|\zeta\rangle_{a}
$$

Since the subspace $\left\langle\left\{|\zeta\rangle_{a}\right\}\right\rangle$ is dense, we obtain

$$
V(g)^{*} a V(g)=\left(\mu^{*} a+\nu\right)\left(\nu^{*} a+\mu\right)^{-1},
$$

where we can define the bounded operator $\left(\nu^{*} a+\mu\right)^{-1}$ by $\left(\nu^{*} a+\mu\right)^{-1}:=\frac{1}{\mu} \sum_{n=1}^{\infty}\left(-\frac{\nu^{*}}{\mu} a\right)^{n}$ because the norm of the operator $-\frac{\nu^{*}}{\mu} a$ is less than 1 .

$+\langle X\rangle$ denotes the vector space whose elements are finite linear sums of a set $X$. 
Next, we consider the action to the operator $A$. Similarly to (36), we have

$$
V(g) A V(g)^{*}|\eta\rangle_{A}=\frac{\left(\mu_{1}+\nu_{1}\right) \eta-\mu_{2}-\nu_{2}}{\left(\mu_{2}-\nu_{2}\right) \eta+\mu_{1}-\nu_{1}}|\eta\rangle_{A},
$$

where we simplifies $\mu_{i} \circ \pi_{\mathrm{SU}(1,1)}(g)$ and $\nu_{i} \circ \pi_{\widehat{\mathrm{SU}(1,1)}}(g)$ as $\mu_{i}$ and $\nu_{i}$, respectively.

\section{Concrete representations of $\mathfrak{s u}(1,1)$}

\subsection{Representation associated with irreducible unitary representation of affine group}

Next, we will construct lowest-weight-type irreducible unitary representations of the algebra $\mathfrak{s u}(1,1)$ from an irreducible unitary representation of the affine group $(a x+b$ group) generated by $E_{+}$and $E_{0}$. The representation which will be constructed in this section is closely related to the continuous wavelet transformation [26, 27]. In this representation, the pair $A$ and $|\eta\rangle_{A}$ plays a more important role than the pair $a$ and $|\zeta\rangle_{a}$. According to Aslaksen and Klauder [28], there is not any irreducible representation of the affine group but the representations equivalent unitarily to the following representation on $L^{2}\left(\mathbb{R}^{+}\right)$or $L^{2}\left(\mathbb{R}^{-}\right)$;

$$
E_{0}=i(P Q+Q P), \quad E_{+}=i Q,
$$

where $E_{0}$ and $E_{+}$are shown to be skew adjoint. In this representation, the vector $\sqrt{\frac{(2 \operatorname{Im} \eta)^{2 k+1}}{\Gamma(2 k+1)}} x^{k} e^{i \eta x}$ is called the affine coherent state , and it is obtained by operating the affine group on the affine vacuum state $\sqrt{\frac{2^{2 k+1}}{\Gamma(2 k+1)}} x^{k} e^{\mp x}$. In the following, we will construct an irreducible unitary representation of the algebra $\mathfrak{s u}(1,1)$ from the above type of unitary representation of the affine group, and will discuss how to interpret the affine coherent states in terms of the unitary representation of the algebra $\mathfrak{s u}(1,1)$. Therefore, in addition to the two generators in (37), we should introduce the representation of another additional generator $E_{-}$. By choosing

$$
\tilde{E}_{-, k}:=-i\left(P Q P+k^{2} Q^{-1}\right) \quad(k>-1 / 2)
$$

for this additional generator, we can construct an irreducible unitary representation where the triplet $E_{0}, E_{+}$and $E_{-}$satisfies the commutation relations (11). However, we should be careful about the domain of $\tilde{E}_{-, k}$, as follows; first, define the dense subspace $\mathcal{D}_{o}\left(\tilde{E}_{-, k}\right)$ of $L^{2}\left(\mathbb{R}^{+}\right)$by

$\mathcal{D}_{o}\left(\tilde{E}_{-, k}\right)$

$:=\left\{\begin{array}{l|l}f(x)=x^{k} f_{0}(x) \in L^{2}\left(\mathbb{R}^{+}\right) \cap C^{1}\left(\mathbb{R}^{+}\right) \mid \begin{array}{l}(2 k+1) x^{k} f_{0}^{\prime}(x)+x^{k+1} f_{0}^{\prime \prime}(x) \in L^{2}\left(\mathbb{R}^{+}\right), \\ \limsup _{s \rightarrow 0} f_{0}(s)<\infty, \quad x^{k} f_{0}(x) \rightarrow 0 \text { as } x \rightarrow \infty\end{array}\end{array}\right\}$.

Then $\tilde{E}_{-, k}$ is an operator defined on $\mathcal{D}_{o}\left(\tilde{E}_{-, k}\right)$. We need attention to the domain when $-\frac{1}{2}<k<\frac{1}{2}$.

* The Fourier transform of this affine coherent state is equivalent to the Cauchy wavelet in signal processing, whose basic wavelet function is $\frac{\text { (const.) }}{(t \pm i)^{k+1}}$. 
Subnormal operators regarded as generalized observables

Lemma 40 The operator $\tilde{E}_{-, k}$ has the skew-adjoint extension, uniquely.

In the following, its skew-adjoint extension is written by $E_{-, k}$.

Proof It is confirmed that $i \tilde{E}_{-, k}=P Q P+k^{2} Q^{-1}$ is a symmetric operator on $\mathcal{D}_{o}\left(\tilde{E}_{-, k}\right)$, from the fact that the difference

$$
\begin{aligned}
\int_{s}^{t}((P Q P+ & \left.\left.k^{2} Q^{-1}\right) f\right)^{*}(x) g(x) d x-\int_{s}^{t}(f(x))^{*}\left(\left(P Q P+k^{2} Q^{-1}\right) g\right)(x) d x \\
= & {\left[x\left(f^{\prime}(x)\right)^{*} g(x)-x g^{\prime}(x)(f(x))^{*}\right]_{s}^{t} } \\
= & t\left(f^{\prime}(t)\right)^{*} g(t)-t g^{\prime}(t)(f(t))^{*} \\
& -\left(s g(s)\left(\left(f^{\prime}(s)\right)^{*}-\frac{k}{s}(f(s))^{*}\right)-s(f(s))^{*}\left(g^{\prime}(s)-\frac{k}{s} g(s)\right)\right) \\
= & t\left(f^{\prime}(t)\right)^{*} g(t)-t g^{\prime}(t)(f(t))^{*}-\left(s g(s) s^{k}\left(f_{0}^{\prime}(s)\right)^{*}-s(f(s))^{*} s^{k} g_{0}^{\prime}(s)\right)
\end{aligned}
$$

tends to zero as $s \rightarrow 0, t \rightarrow \infty$. Since $i \tilde{E}_{-, k}$ is semi-bounded, Friedrich extension theorem guarantees that there uniquely exists the self-adjoint extension of $i \tilde{E}_{-, k}$. (See pp. 177 of Reed and Simon [29].) Now, the proof is complete.

By letting $L_{+, k}, L_{-, k}, L_{0, k}, \tilde{A}_{k}, A_{k}, N_{k},|n\rangle_{N}^{k}$ and $|\eta\rangle_{A}^{k}$ be $L_{+}, L_{-}, L_{0}, \tilde{A}, A, N,|n\rangle_{N}$ and $|\eta\rangle_{A}$ in this representation, respectively, we have

$$
\begin{aligned}
& L_{+, k}=\frac{1}{2}\left(i(P Q+Q P)-Q+P Q P+k^{2} Q^{-1}\right), \\
& L_{-, k}=\frac{1}{2}\left(i(P Q+Q P)+Q-P Q P-k^{2} Q^{-1}\right), \\
& L_{0, k}=\left(P Q P+k^{2} Q^{-1}+Q\right), \quad \tilde{A}_{k}=P+i k Q^{-1}, \\
& N_{k}=\frac{1}{2}\left(P Q P+k^{2} Q^{-1}+Q-1-2 k\right), \\
& |n\rangle_{N}^{k}(x)=\sqrt{\frac{2^{2 k+1} n !}{\Gamma(n+2 k+1)}} e^{-x} x^{k} S_{n}^{2 k}(2 x), \\
& |\eta\rangle_{A}^{k}(x)=\sqrt{\frac{(2 \operatorname{Im} \eta)^{2 k+1}}{\Gamma(2 k+1)}} x^{k} e^{i \eta x},
\end{aligned}
$$

when $S_{n}^{l}(x)$ is the Sonine Polynomial (or the associated Laguerre polynomial) defined by困

$$
S_{n}^{l}(x):=\sum_{m=0}^{n} \frac{(-1)^{m}}{(n-m) !} \frac{\Gamma(n+l+1) x^{m}}{\Gamma(m+l+1) m !} .
$$

$|\eta\rangle_{A}^{k}(x)$ is the affine coherent state. Moreover, in this representation, the minimum eigenvalue of $L_{0, k}$ is $\lambda=2 k+1$, and the Casimir operator is $4 k^{2}-1$. Thus, we have the following theorem.

Theorem 41 The representations of lowest-weight-type (defined in section 4 ) in general can be concretely constructed by (37) and (38) on $L^{2}\left(\mathbb{R}^{+}\right)$in the correspondence $\lambda=2 k+1$.

$\sharp$ Sometimes another definition with $n+l$ instead of $l$ is used. 
Remark 6 [Domains of $A_{k}, A_{k}^{*}$ ] In the following, we will show the properties of $\tilde{A}_{k}$ in order to show the properties of $\tilde{A}$ in the representations of lowest-weight-type. Since the domain of $\tilde{A}_{k}$ is $\left\langle\{|n\rangle\}_{n=0}^{\infty}\right\rangle$ and $\tilde{A}_{k}=P+i k Q^{-1}$, the relation

$\mathcal{D}_{o}\left(\tilde{A}_{k}^{*}\right) \cap C^{1}\left(\mathbb{R}^{+}\right)=\left\{\begin{array}{l|l}x^{-k} f(x) \in L^{2}\left(\mathbb{R}^{+}\right) \cap C^{1}\left(\mathbb{R}^{+}\right) & \begin{array}{l}x^{-k} f^{\prime}(x) \in L^{2}\left(\mathbb{R}^{+}\right) \\ f(s) \rightarrow 0 \text { as } s \rightarrow 0,\end{array}\end{array}\right\}$

is derived, and hence we can show that $\mathcal{D}_{o}\left(\tilde{A}_{k}^{*}\right)$ is dense in $L^{2}\left(\mathbb{R}^{+}\right)$. Thus $\tilde{A}_{k}$ is shown to be a closable operator. Since $A_{k}=\overline{\tilde{A}_{k}}$, the relation

$\mathcal{D}_{o}\left(A_{k}\right) \cap C^{1}\left(\mathbb{R}^{+}\right)=\left\{\begin{array}{l|l}x^{k} f(x) \in L^{2}\left(\mathbb{R}^{+}\right) \cap C^{1}\left(\mathbb{R}^{+}\right) & \begin{array}{l}x^{k} f^{\prime}(x) \in L^{2}\left(\mathbb{R}^{+}\right), \\ \limsup _{s \rightarrow 0} f(s)<\infty\end{array}\end{array}\right\}$.

is confirmed. Note that $\bar{X}=X^{* *}$ and $X^{*}=\bar{X}^{*}$ hold for a densely defined linear operator $X$, and that $\lim \sup _{x \rightarrow \infty} f_{0}(x)=0$ for $k>-\frac{1}{2}$ when $x^{k} f_{0}(x) \in L^{2}\left(\mathbb{R}^{+}\right)$. From (40), we can show that $|\zeta\rangle_{a} \in \mathcal{D}_{o}\left(A_{k}\right)$. Thus, the subspaces $\mathcal{D}_{o}\left(A_{k}^{*}\right) \cap C^{1}\left(\mathbb{R}^{+}\right)$and $\mathcal{D}_{o}\left(A_{k}\right) \cap C^{1}\left(\mathbb{R}^{+}\right)$are the cores $\mathbb{\text { Of }}$ of $A_{k}^{*}$ and $A_{k}$. When $-\frac{1}{2}<k<\frac{1}{2}$, the domain of $A_{k}$ is larger than the domain of $A_{-k}^{*}$, though $A_{k}$ and $A_{-k}^{*}$ are the same formally, i.e. $A_{-k}^{*} \varsubsetneqq A_{k}$. In the special case where $\lambda=1$ (i.e. where $k=0$ ), $A_{0}^{*}$ is symmetric. Since $A_{0}=A_{0}^{* *}$ has no spectrum in the lower half plane, $A_{0}^{*}$ 's deficiency indices are $(1,0)$. (For the definition of deficiency indices, see p.138 of Reed and Simon [29] or p.360 of Rudin [11].) Therefore, the operator $A_{0}^{*}$ is a maximal symmetric operator.

These relations $\mathcal{D}_{o}\left(A_{k}^{*}\right) \subset \mathcal{D}_{o}\left(A_{k}\right)$ and $\mathcal{D}_{o}\left(A_{k}^{*}\right) \subset \mathcal{D}_{o}\left(E_{+}^{-1}\right)$ are shown in the cases where $\lambda<1(k>0)$, only the relation $\mathcal{D}_{o}\left(A_{k}^{*}\right) \subset \mathcal{D}_{o}\left(A_{k}\right)$ is shown when $\lambda=1(k=0)$, and these relations $\mathcal{D}_{o}\left(A_{k}\right) \subset \mathcal{D}_{o}\left(A_{k}^{*}\right)$ and $\mathcal{D}_{o}\left(A_{k}\right) \subset \mathcal{D}_{o}\left(E_{+}^{-1}\right)$ are shown when $0<\lambda<1\left(-\frac{1}{2}<k<0\right)$. From Theorem [6, these discussions and (28), we obtain $(30)$.

\subsection{Representation associated with squeezed states}

Next, we will discuss the following representation of the algebra $\mathfrak{s u}(1,1)$ on the Hilbert space $L^{2}(\mathbb{R})$; let

then we have

$$
E_{0}=\frac{i}{2}(P Q+Q P), \quad E_{+}=\frac{i}{2} Q^{2}, \quad E_{-}=-\frac{i}{2} P^{2}
$$

$$
L_{0}=n_{b}+\frac{1}{2}, \quad L_{+}=-\frac{1}{2}\left(a_{b}^{*}\right)^{2}, \quad L_{-}=\frac{1}{2}\left(a_{b}\right)^{2},
$$

where the boson annihilation operator $a_{b}$ and the boson number operator $n_{b}$ are given by $a_{b}=\sqrt{\frac{1}{2}}(Q+i P)$ and $n_{b}=\frac{1}{2}\left(Q^{2}+P^{2}-1\right)=a_{b}^{*} a_{b}$. In this representation, the Casimir operator is the scalar $-\frac{3}{4}$. From the fact that the Casimir operator is the scalar $\lambda(\lambda-2)$, the solutions are $\lambda=1 / 2,3 / 2$. Under the representation given in (41), $L^{2}(\mathbb{R})$ is not irreducible and it is decomposed into two irreducible subspaces as:

$$
L^{2}(\mathbb{R})=L_{\text {even }}^{2}(\mathbb{R}) \oplus L_{\text {odd }}^{2}(\mathbb{R})
$$

$\dagger \dagger$ A subspace of the domain $\mathcal{D}_{o}(X)$ of a closed operator $X$ is called a core of $X$ if it is dense in $\mathcal{D}_{o}(X)$ with respect to the graph norm of the operator $X$. 
where $L_{\text {even }}^{2}(\mathbb{R})$ is the set of square-integrable even functions and $L_{\text {odd }}^{2}(\mathbb{R})$ is the set of square-integrable odd functions. The solution $\lambda=1 / 2$ corresponds to the subspace $L_{\text {even }}^{2}(\mathbb{R})$ and the solution $\lambda=3 / 2$ does to the subspace $L_{\text {odd }}^{2}(\mathbb{R})$. In the subspace $L_{\text {even }}^{2}(\mathbb{R})$, the operators $a, A$ and $N$ are written in the forms

$$
\begin{aligned}
& a=-\left(a_{b}^{*}\right)^{-1} a_{b}, \quad A=Q^{-1} P, \quad N=\frac{1}{2} n_{b}, \quad|n\rangle_{N}=(-1)^{n}|2 n\rangle_{n_{b}}, \\
& \mathcal{D}_{o}(A) \cap C^{1}(\mathbb{R})=\left\{f \in L_{\text {even }}^{2}(\mathbb{R}) \cap C^{1}(\mathbb{R}) \mid \frac{1}{x} f^{\prime}(x) \in L^{2}(\mathbb{R})\right\},
\end{aligned}
$$

where $|n\rangle_{n_{b}}$ denotes the eigenvector in $L^{2}(\mathbb{R})$ of the boson number operator $n_{b}$ associated with the eigenvalue $n$.

Lemma 42 In the action of $\mathfrak{s u}(1,1)$ on $L_{\text {even }}^{2}(\mathbb{R})$, we have

$$
|0 ; \mu, \nu\rangle\langle 0 ; \mu, \nu|=V(g)| 0\rangle_{a}{ }_{a}\left\langle 0\left|V(g)^{*}=\right| \frac{\nu}{\mu}\right\rangle_{a}{ }_{a}\left\langle\frac{\nu}{\mu}|=| i \frac{\mu+\nu}{\mu-\nu}\right\rangle_{A}{ }_{A}\left\langle i \frac{\mu+\nu}{\mu-\nu}\right| .
$$

Remark that the squeezed state $|0 ; \mu, \nu\rangle$ is defined as the unit eigen vector of $b_{\mu, \nu}=$ $\mu a_{b}+\nu a_{b}^{*}$ associated with the eigen value 0 .

Proof We need the discussion of Remark 3 and 5 for the proof. It is necessary to discuss the action of the group. From this representation of the algebra $\mathfrak{s u}(1,1)$, we can construct the representation of the double-covering group $\widehat{\mathrm{SU}(1,1)}$ of the group $\mathrm{SU}(1,1)$. In general, we can construct the representation of $\widehat{\mathrm{SU}(1,1)}$ in the case where $\lambda$ is a half-integer. Now, we let $\widetilde{\mathrm{SU}_{(1,1)}}$ be the projection from $\mathrm{SU}(1,1)$ to $\mathrm{SU}(1,1)$. From (41), we have

$$
\begin{aligned}
& e^{t E_{0}} Q e^{-t E_{0}}=e^{t} Q, \quad e^{t E_{+}} Q e^{-t E_{+}}=Q, \quad e^{t E_{-}} Q e^{-t E_{-}}=Q-P t, \\
& e^{t E_{+}} P e^{-t E_{+}}=P-Q t, \quad e^{t E_{0}} P e^{-t E_{0}}=e^{-t} P, \quad e^{t E_{0}} P e^{-t E_{0}}=P .
\end{aligned}
$$

From (31), (32) and some calculations, we have

$$
\begin{aligned}
& V(g) Q V(g)^{*}=\left(\mu_{1}+\nu_{1}\right) Q+\left(\nu_{2}-\mu_{2}\right) P, \\
& V(g) P V(g)^{*}=\left(\nu_{2}+\mu_{2}\right) Q+\left(\mu_{1}-\nu_{1}\right) P, \quad \forall g \in \widehat{\mathrm{SU}(1,1)},
\end{aligned}
$$

where the complex numbers $\mu_{i} \circ \pi_{\mathrm{SU}(1,1)}(g)$ and $\nu_{i} \circ \pi_{\mathrm{SU}(1,1)}(g)$ with the functions $\mu_{i}$ and $\nu_{i}$ defined at the beginning of Remark 3 are denoted simply by $\mu_{i}$ and $\nu_{i}$, respectively, in a similar manner to the previous section. Thus, we have

$$
\begin{aligned}
& V(g) a_{b} V(g)^{*}=\mu a_{b}+\nu a_{b}^{*}, \\
& V(g) a_{b}^{*} V(g)^{*}=\nu^{*} a_{b}+\mu^{*} a_{b}^{*}, \quad \forall g \in \widehat{\mathrm{SU}(1,1)},
\end{aligned}
$$

where we simplifies $\mu \circ \pi_{\mathrm{SU}(1,1)}(g)$ and $\nu \circ \pi_{\mathrm{SU}(1,1)}(g)$ by $\mu$ and $\nu$, respectively, similarly.

Since $L_{-}=\frac{1}{2}\left(a_{b}\right)^{2}$, the lowest weight vector $|0\rangle_{a}$ is the boson vacuum vector $|0 ; 1,0\rangle$. The squeezed state $|0 ; \mu, \nu\rangle$ satisfies $\left(\mu a_{b}+\nu a_{b}^{*}\right)|0 ; \mu, \nu\rangle=0$. Assume that $\mu \circ \pi_{\mathrm{SU}(1,1)}(g)=\mu, \nu \circ \pi_{\mathrm{SU}(1,1)}(g)=\nu$. Then we have $V(g) a_{b} V(g)^{*}|0 ; \mu, \nu\rangle=0$. Hence, we see that the vector $V(g)^{*}|0 ; \mu, \nu\rangle$ equals a scalar-times vacuum vector $|0 ; 1,0\rangle=|0\rangle_{a}$. From these facts and (34), we obtain (43). 
From (43), we find the correspondence to the characteristic equations (11) and (2) of squeezed states explained in section 11. Substituting (43) into (24) and (27), we obtain (2) and (1). In the following, the vector $|\zeta\rangle_{a}$ in $L_{\text {even }}^{2}(\mathbb{R})$ is denoted by $|\zeta\rangle_{a \text {,even. The }}$ equations (23) and (43) implies that the squeezed state $|0 ; \mu, \nu\rangle$ equals a scalar-times $\exp \left(-\frac{\xi}{2}\left(a_{b}^{*}\right)^{2}+\frac{\xi^{*}}{2}\left(a_{b}\right)^{2}\right)|0 ; 1,0\rangle$ corresponding to Caves's notation 30 of squeezed state, where $\xi:=\frac{1}{2} e^{i \arg \frac{\nu}{\mu}} \ln \frac{|\mu|+|\nu|}{|\mu|-|\nu|}$.

On the other hand, in $L_{\text {odd }}^{2}(\mathbb{R})$, the operators $a, A$ and $N$ are written in the forms

$$
a=-a_{b}\left(a_{b}^{*}\right)^{-1}, \quad A=P Q^{-1}, \quad N=\frac{1}{2}\left(n_{b}-1\right), \quad|n\rangle_{N}=(-1)^{n}|2 n+1\rangle_{n_{b}} .
$$

Next, we will discuss the representation of the algebra $\mathfrak{s u}(1,1)$ in the Hilbert space $L^{2}\left(\mathbb{R}^{n}\right)=\underbrace{L^{2}(\mathbb{R}) \otimes \cdots \otimes L^{2}(\mathbb{R})}_{n}$, for multi-particle systems. In this representation,

$$
E_{0}=\frac{i}{2} \sum_{j=1}^{n}\left(P_{j} Q_{j}+Q_{j} P_{j}\right), \quad E_{+}=\frac{i}{2} \sum_{j=1}^{n} Q_{j}^{2}, \quad E_{-}=-\frac{i}{2} \sum_{j=1}^{n} P_{j}^{2},
$$

hold, where $Q_{j}$ and $P_{j}$ denotes the multiplication operator and the $(-i)$-times differential operator, respectively, with respect to the $j$-th variable. Let $L_{\mathrm{e}}^{2}\left(\mathbb{R}^{n}\right)$ be the closure of the linear space generated by $\{|\zeta\rangle_{a, \text { even }}^{\otimes n}:=\underbrace{|\zeta\rangle_{a, \text { even }} \otimes \cdots \otimes|\zeta\rangle_{a, \text { even }}}_{n}\}$. Then, the Hilbert space $L_{\mathrm{e}}^{2}\left(\mathbb{R}^{n}\right)$ is irreducible under the representation (44) of the algebra $\mathfrak{s u}(1,1)$, and then we have $L_{\mathrm{e}}^{2}\left(\mathbb{R}^{n}\right)=\left\{f \in L^{2}\left(\mathbb{R}^{n}\right) \mid f\right.$ is a function of $\left.\sum_{j=1}^{n} x_{j}^{2}\right\}$, and then the vector $|\zeta\rangle_{a}$ in this representation on $L_{\mathrm{e}}^{2}\left(\mathbb{R}^{n}\right)$ is equivalent to $|\zeta\rangle_{a, \text { even }}^{\otimes n}$.

Letting $A_{n, \mathrm{e}}$ denote the operator $A$ in this representation, we obtain the relation

$$
A_{n, \mathrm{e}}=\left(\sum_{j=1}^{n} Q_{j}^{2}\right)^{-1} \sum_{j=1}^{n} Q_{j} P_{j}=-i\left(\sum_{j=1}^{n} \frac{2 x_{j}}{r} \frac{\partial}{\partial x_{j}}\right)
$$

with $r:=2 \sum_{j=1}^{n} x_{j}^{2}$. Now define the unitary map $U_{n}: L^{2}\left(\mathbb{R}^{n}\right) \rightarrow L^{2}\left(\mathbb{R}^{+}\right) \otimes L^{2}\left(S^{n-1}\right) \cong$ $L^{2}\left(\mathbb{R}^{+} \times S^{n-1}\right)$ by $\left(U_{n}(f)\right)\left(r,\left(e_{1}, e_{2}, \ldots, e_{n}\right)\right)=r^{\frac{n-2}{4}} f\left(\sqrt{\frac{r}{2}} e_{1}, \sqrt{\frac{r}{2}} e_{2}, \ldots, \sqrt{\frac{r}{2}} e_{n}\right)$, where $S^{n-1}$ denotes the $(n-1)$-dimensional spherical surface and $\left(e_{1}, e_{2}, \ldots, e_{n}\right)$ is an element of $S^{n-1}$. Then, the following relations hold;

$$
\begin{aligned}
& U_{n} E_{0} U_{n}^{*}=E_{0, \frac{n-2}{4}} \otimes I, \quad U_{n} E_{+} U_{n}^{*}=E_{+, \frac{n-2}{4}} \otimes I, \quad U_{n} E_{-} U_{n}^{*}=E_{-, \frac{n-2}{4}} \otimes I, \\
& U_{n} A_{n, \mathrm{e}} U_{n}^{*}=\left(P+i\left(\frac{n}{4}-\frac{1}{2}\right) Q^{-1}\right) \otimes I=-i \frac{\partial}{\partial r}+i\left(\frac{n}{4}-\frac{1}{2}\right) \frac{1}{r}, \\
& U_{n} L_{\mathrm{e}}^{2}\left(\mathbb{R}^{n}\right)=L^{2}\left(\mathbb{R}^{+}\right) \otimes \psi_{n}, \\
& U_{n} \mathcal{D}_{o}\left(A_{n, \mathrm{e}}\right) \cap\left(C^{1}\left(\mathbb{R}^{+}\right) \otimes \psi_{n}\right) \\
& \quad=\left\{\begin{array}{l|l}
x^{\frac{n}{4}-\frac{1}{2}} f(x) \in L^{2}\left(\mathbb{R}^{+}\right) \cap C^{1}\left(\mathbb{R}^{+}\right) & \begin{array}{l}
x^{\frac{n}{4}-\frac{1}{2}} f^{\prime}(x) \in L^{2}\left(\mathbb{R}^{+}\right) \\
f(s)<\infty \text { as } s \rightarrow 0 .
\end{array}
\end{array}\right\} \otimes \psi_{n},
\end{aligned}
$$

where $\psi_{n}$ denotes the constant function on $S^{n-1}$ such that $\left\|\psi_{n}\right\|=1$. The compoundsystem-type normal extension of $A_{n, \mathrm{e}}$ in the above relations is reduced to the discussion of $A_{\frac{n}{4}-\frac{1}{2}}$ which will be treated in section 6.1 and section 6.2 . 
Subnormal operators regarded as generalized observables

\section{Construction of compound-system-type normal extension of $A^{*}$}

\subsection{The case where $\lambda=1$}

In this subsection, we will construct an compound-system-type normal extension of $A^{*}$ when $\lambda=1$. Let $\{|\uparrow\rangle,|\downarrow\rangle\}$ be a CONS of $\mathbb{C}^{2}$. From Lemma 28 and the fact that $A^{*}$ is maximal symmetric, we obtain the following theorem.

Theorem 43 Define the operator $T:=A \otimes|-\rangle\left\langle+\left|+A^{*} \otimes\right|+\right\rangle\langle-|$ on the domain $\mathcal{D}_{o}(T):=\mathcal{D}_{o}(A) \otimes|+\rangle \oplus \mathcal{D}_{o}\left(A^{*}\right) \otimes|-\rangle$ with $| \pm\rangle:=\frac{1}{\sqrt{2}}(|\uparrow\rangle \pm|\downarrow\rangle)$. The operator $T$ is a self-adjoint operator. Moreover, the triple $\left(\mathbb{C}^{2}, T,|\uparrow\rangle\right)$ is a compound-system-type normal extension of $A^{*}$.

Similarly, we can construct a compound-system-type normal extension of $a^{*}$ according to Lemma 27. The spectrum of the compound-system-type normal extension of $A^{*}$ for $\lambda=1$ appears only on the real axis. That of the compound-system-type normal extension of $a^{*}$ appears only on the unit circle.

\subsection{The cases where $\lambda>1$}

In the following, we will discuss the cases when $\lambda>1$. Let $\{|\uparrow\rangle,|\downarrow\rangle\}$ be CONS of $\mathbb{C}^{2}$. We obtain the following theorem, with $A_{0}\left(A_{k}\right.$ with $\left.k=0\right)$ discussed at the end of section 5.1.

Theorem 44 The pair of $E_{+} \otimes I$ and $E_{0} \otimes I+I \otimes E_{0}$ on $\mathcal{H}_{\lambda} \otimes \mathcal{H}_{\lambda-1}$ satisfies the commutation relation of the generators of Affine group. This representation of Affine group is written as follows; there exist a Hilbert space $\mathcal{H}^{\prime}$ and a unitary map $U$ from $\mathcal{H}_{\lambda} \otimes \mathcal{H}_{\lambda-1}$ to $\mathcal{H}^{\prime} \otimes L^{2}\left(\mathbb{R}^{+}\right)$such that $U\left(E_{+} \otimes I\right) U^{*}=I \otimes E_{+}, U\left(E_{0} \otimes I+I \otimes E_{0}\right) U^{*}=I \otimes E_{0}$. Then, the operator $U^{*}\left(I \otimes A_{0}\right) U \otimes|-\rangle\left\langle+\left|+U^{*}\left(I \otimes A_{0}^{*}\right) U \otimes\right|+\right\rangle\langle-|$ with the domain $\mathcal{D}_{o}\left(U^{*}\left(I \otimes A_{0}\right) U\right) \otimes|+\rangle \oplus \mathcal{D}_{o}\left(U^{*}\left(I \otimes A_{0}\right) U\right) \otimes|-\rangle$ is self-adjoint.

Moreover, the operator $T:=U^{*}\left(I \otimes A_{0}\right) U \otimes|-\rangle\left\langle+\left|+U^{*}\left(I \otimes A_{0}^{*}\right) U \otimes\right|+\right\rangle\langle-|-i E_{+}^{-1} \otimes$ $E_{+} \otimes I$ with the domain $\mathcal{D}_{o}(T):=\left(\mathcal{D}_{o}\left(U^{*}\left(I \otimes A_{0}\right) U\right) \otimes|+\rangle \oplus \mathcal{D}_{o}\left(U^{*}\left(I \otimes A_{0}^{*}\right) U\right) \otimes|-\rangle\right) \cap$ $\mathcal{D}_{o}\left(E_{+}^{-1} \otimes E_{+}\right) \otimes \mathbb{C}^{2}$ is normal. The triple $\left(\mathcal{H}_{\lambda}^{\prime}:=\mathcal{H}_{\lambda-1} \otimes \mathbb{C}^{2}, T, \psi:=|0\rangle_{N} \otimes|\uparrow\rangle\right)$ is a compound-system-type normal extension of $A^{*}$.

Proof We need the discussion of Remark 6 for the proof. It is sufficient to prove them under the representations given in section 5.1 because of Theorem 41. Now define the unitary operator $U$ on $L^{2}\left(\mathbb{R}^{+}\right) \otimes L^{2}\left(\mathbb{R}^{+}\right)$by $(U(f))(u, v)=\sqrt{v} f(v, u v)$. Then we have $U\left(E_{+} \otimes I\right) U^{*}=I \otimes E_{+}, U\left(E_{0} \otimes I+I \otimes E_{0}\right) U^{*}=I \otimes E_{0}$ and $U\left(-i E_{+}^{-1} \otimes E_{+}\right) U^{*}=-E^{+} \otimes I$. Because the discussion at the end of section 5.1 shows that $A_{0}$ is closed and symmetric, it follows from the proof of Lemma 28 that the operator $A_{0} \otimes|-\rangle\left\langle+\left|+A_{0}^{*} \otimes\right|+\right\rangle\langle-|$ is self-adjoint and its domain is $\mathcal{D}_{o}\left(A^{*}\right) \otimes|-\rangle \oplus \mathcal{D}_{o}(A) \otimes|+\rangle$. In general, for a self-adjoint operator $X$ on $\mathcal{K}_{1}$ and a skew-adjoint operator $Y$ on $\mathcal{K}_{2}$, we can show that the operator $X \otimes I+I \otimes Y$ with the domain $\mathcal{D}_{o}(X) \otimes \mathcal{D}_{o}(Y)=\mathcal{D}_{o}(X) \otimes \mathcal{K}_{2} \cap \mathcal{K}_{1} \otimes \mathcal{D}_{o}(Y) \subset \mathcal{K}_{1} \otimes \mathcal{K}_{2}$ is normal. Then, the operator $T^{\prime}:=I \otimes\left(A_{0} \otimes|-\rangle\left\langle+\left|\oplus A_{0}^{*} \otimes\right|+\right\rangle\langle-|\right)-E^{+} \otimes I \otimes I$ with the domain $\mathcal{D}_{o}\left(T^{\prime}\right):=\left(\mathcal{D}_{o}\left(I \otimes A_{0}\right) \otimes|+\rangle \oplus \mathcal{D}_{o}\left(I \otimes A_{0}^{*}\right) \otimes|-\rangle\right) \cap \mathcal{D}_{o}\left(E_{+}\right) \otimes L^{2}\left(\mathbb{R}^{+}\right) \otimes \mathbb{C}^{2}$ 
is normal. Thus, we have proved that the operator $T\left(=U^{*} T^{\prime} U\right)$ is normal. Now, we will prove that the triple $\left(\mathcal{H}_{\lambda}^{\prime}, T, \psi=|0\rangle_{N}^{k-\frac{1}{2}} \otimes|\uparrow\rangle\right)$ is a compound-system-type normal extension of $A_{k}^{*}$. Since the set $\mathcal{D}_{o}\left(A_{k}^{*}\right) \cap C^{1}\left(\mathbb{R}^{+}\right)$is a core of the operator $A_{k}^{*}$, it is sufficient to show that $\left(A_{k}^{*} \phi\right) \otimes|0\rangle_{N}^{k-\frac{1}{2}} \otimes|\uparrow\rangle=T\left(\phi \otimes|0\rangle_{N}^{k-\frac{1}{2}} \otimes|\uparrow\rangle\right)$ for any $\phi \in \mathcal{D}_{o}\left(A_{k}^{*}\right) \cap C^{1}\left(\mathbb{R}^{+}\right)$.

From the definitions and (39), some calculations result in

$$
\begin{aligned}
U\left(\left(\mathcal{D}_{o}\left(A_{k}^{*}\right)\right.\right. & \left.\left.\cap C^{1}\left(\mathbb{R}^{+}\right)\right) \otimes|0\rangle_{N}^{k-\frac{1}{2}}\right) \\
& =\left\{f(v) u^{k-1 / 2} e^{-u v} \in L^{2}\left(\mathbb{R}^{+} \times \mathbb{R}^{+}\right) \mid x^{-k} f^{\prime}(x) \in L^{2}\left(\mathbb{R}^{+}\right), f(s) \rightarrow 0 \text { as } s \rightarrow 0\right\} .
\end{aligned}
$$

We can show that a function $u \mapsto u^{k-1 / 2} e^{-u v}$ is contained by $\mathcal{D}_{o}\left(E_{+}\right) \subset L^{2}\left(\mathbb{R}^{+}\right)$for any $v \in \mathbb{R}^{+}$. If a function $f$ satisfies the condition $x^{-k} f^{\prime}(x) \in L^{2}\left(\mathbb{R}^{+}\right), f(s) \rightarrow 0$ as $s \rightarrow 0$, then a function $v \mapsto f(v) u^{k-1 / 2} e^{-u v}$ is contained by $\mathcal{D}_{o}\left(A_{0}^{*}\right) \subset L^{2}\left(\mathbb{R}^{+}\right)$for any $u \in \mathbb{R}^{+}$.

Then, the set $U\left(\left(\mathcal{D}_{o}\left(A_{k}^{*}\right) \cap C^{1}\left(\mathbb{R}^{+}\right)\right) \otimes|0\rangle_{N}^{k-\frac{1}{2}}\right)$ is included in the set $\mathcal{D}_{o}\left(I \otimes A_{0}^{*}\right) \cap \mathcal{D}_{o}\left(E_{+} \otimes I\right) \cap\left(C^{1}\left(\mathbb{R}^{+}\right) \otimes C^{1}\left(\mathbb{R}^{+}\right)\right)$. Hence,

$$
\begin{aligned}
U\left(\left(\mathcal{D}_{o}\left(A_{k}^{*}\right) \cap\right.\right. & \left.\left.C^{1}\left(\mathbb{R}^{+}\right)\right) \otimes|0\rangle_{N}^{k-\frac{1}{2}}\right) \otimes|\uparrow\rangle \\
\subset & \mathcal{D}_{o}\left(I \otimes A_{0}^{*} \otimes I\right) \cap \mathcal{D}_{o}\left(E_{+} \otimes I \otimes I\right) \cap\left(C^{1}\left(\mathbb{R}^{+}\right) \otimes C^{1}\left(\mathbb{R}^{+}\right) \otimes|\uparrow\rangle\right) \\
\subset & \mathcal{D}_{o}\left(I \otimes\left(A_{0}^{*} \otimes|+\rangle\left\langle-\left|+A_{0} \otimes\right|-\right\rangle\langle+|\right)\right) \cap \mathcal{D}_{o}\left(E_{+} \otimes I \otimes I\right) \\
& \cap\left(C^{1}\left(\mathbb{R}^{+}\right) \otimes C^{1}\left(\mathbb{R}^{+}\right) \otimes|\uparrow\rangle\right) \\
= & \mathcal{D}_{o}\left(T^{\prime}\right) \cap\left(C^{1}\left(\mathbb{R}^{+}\right) \otimes C^{1}\left(\mathbb{R}^{+}\right) \otimes|\uparrow\rangle\right) .
\end{aligned}
$$

Thus, for the function $f(x)$ satisfying $\phi(x)=f(x) x^{-k}$, we obtain

$$
\begin{aligned}
T\left(\phi \otimes|0\rangle_{N}^{k-\frac{1}{2}}\right. & \otimes|\uparrow\rangle) \\
& =-i \frac{d}{d v}\left(f(v) u^{k-\frac{1}{2}} e^{-u v}\right) \otimes(|+\rangle\langle-|+|-\rangle\langle+|)|\uparrow\rangle-i f(v) u^{k+\frac{1}{2}} e^{-u v} \otimes|\uparrow\rangle \\
& =-i \frac{d f}{d v}(v) u^{k-\frac{1}{2}} e^{-u v} \otimes|\uparrow\rangle \\
& =\left(A_{k} \phi\right) \otimes|0\rangle_{N}^{k-\frac{1}{2}} \otimes|\uparrow\rangle .
\end{aligned}
$$

The theorem is now immediate.

In the above discussions, it is sufficient only to choose $\mathcal{H}_{\lambda-1}$ instead of $\mathcal{H}_{\lambda}^{\prime}$ in order only to show that the operator $T$ formally satisfies $\left[T, T^{*}\right]=0$ and formally satisfies (10). However, the above definition of $\mathcal{H}_{\lambda}^{\prime}$ is required in order that $T$ may be a normal operator defined in Definition 11.

Since the spectrum of the compound-system-type normal extension of $A^{*}$ for $\lambda=1$ appears only in the upper half plane including the real axis, the spectrum of the compound-system-type normal extension of $a^{*}$ appears only on the unit disk (including the unit circle) if the latter is related to the former by the adjoint of the Cayley transform.

\section{Conclusions}

We have discussed subnormal operators as a class of generalized observables. A POVM of a subnormal operator defined in Definition 13 has little information about its 
implementation. However, in order to describe not only the probability distributions characterized by the POVMs but also a framework of their implementations, we have defined compound-system-type normal extensions in section 3. (The heterodyne measurement known in quantum optics is interpreted as a special case of compoundsystem-type normal extensions.) In these contexts, we have constructed the compoundsystem-type normal extensions of two subnormal operators $a^{*}$ and $A^{*}$ canonically introduced from an irreducible unitary representation of $\mathfrak{s u}(1,1)$, when the minimum eigenvalue $\lambda$ of the generator $L_{0}$ is not less than one. The squeezed states are regarded as the coherent states of the algebra $\mathfrak{s u}(1,1)$, and have been characterized as the eigenvectors of an operator defined in this mathematical framework. The squeezed states in two-particle or multi-particle systems have been interpreted as the eigenvectors of the adjoints $a$ and $A$ of the subnormal operators $a^{*}$ and $A^{*}$. The coherent states of the affine group have been interpreted in the same framework, as well. The squeezed states in one-particle system have been interpreted as the eigenvectors of the operator $a$ and $A$, though the operators $a^{*}$ and $A^{*}$ are not subnormal and their compound-system-type normal extensions do not exist in this case because $\lambda$ is less than one in this case.

The information described by a compound-system-type normal extension isn't enough to completely specify the experimental implementation, where the measurement of the normal operator on the compound system is performed by the measurement on each system after some interactions were made between the basic system and the ancillary system. Therefore, the formulation including this specification is one of future problems. As another possibility, since the affine group is closely related to Poincaré group, our results about the affine group may be applicable to the relativistic quantum mechanics.

\section{Acknowledgments}

The authors would like to express our thanks to Dr. S. Shirai, Dr. T. Mine and Mr. M. Miyamoto for their advice and comments on functional analysis. They are indebted to Professor M. Ozawa for suggesting the references [8, 16]. They thank Professor H. Nagaoka for useful discussions. They are also grateful to thank the third referee for useful comments. They also thank Professor F. Hiai for suggesting the originality of the bounded version of Lemma 12.

\section{Appendix}

The following lemma about spectra is well-known. (See Hiai and Yanagi [15].) In Hiai and Yanagi [15], it is proved in the case of bounded operators. But, it can be easily extended to the case of unbounded operators.

Lemma 45 For a densely defined operator $A$ on $\mathcal{H}$, Let $\sigma_{p}(A), \sigma_{c}(A)$ and $\sigma_{r}(A)$, be the point spectrum, the continuous spectrum and the residual spectrum, respectively. Then we have the following relations: 
- $\lambda \in \sigma_{r}(A) \Rightarrow \lambda^{*} \in \sigma_{p}\left(A^{*}\right)$

- $\lambda \in \sigma_{p}(A) \Rightarrow \lambda^{*} \in \sigma_{r}\left(A^{*}\right) \cup \sigma_{p}\left(A^{*}\right)$

- $\lambda \in \sigma_{c}(A) \Rightarrow \lambda^{*} \in \sigma_{c}\left(A^{*}\right)$.

\section{References}

[1] Yuen H P and Lax M 1973 Trans. IEEE IT-19 740.

[2] Halmos P R 1950 Summa Bras. Math. 2125.

[3] Halmos P R 1952 Ann. Polon. Math. 2543.

[4] Perelomov A 1986 Generalized Coherent States and Their Applications (New York: Springer).

[5] Yuen H P 1976 Phys. Rev. A 132226.

[6] Stochel J and Szafraniec F H 1985 J. Operator Theory 1431.

[7] Stochel J and Szafraniec F H 1989 Publ. RIMS, Kyoto Univ. 25105.

[8] Szafraniec F H 2000 Com. Math. Phys. 210323.

[9] Ôta S 1998 Bull. Polish Acad. Sci. Math. 46291.

[10] Lahti P, Pellonpää J P and Ylinen K 1999 J. Math. Phys. 402181.

[11] Rudin W 1991 Functional Analysis 2nd ed. (Boston: McGraw-Hill).

[12] Ozawa M 1980 Rep. Math. Phys. 1811.

[13] Holevo A S 1980 Probabilistic and Statistical Aspects of Quantum Theory (Moscow: Nauka) (In Russian) 1982 (Amsterdam: North-Holland) (English edition).

[14] Naïmark M A 1943 Comptes Rendus (Doklady) de l'Acadenie des Sience de l'URSS 41359.

[15] Hiai F and Yanagi K 1995 Hilbert spaces and linear operators (Tokyo: Makino-Shoten) (In Japanese).

[16] Riesz F and Sz.-Nagy B 1960 Functional analysis 2nd ed. (New York: Ungar) (In French) 1990 (New York: Dover) (English edition).

[17] Helstrom C W 1976 Quantum Detection and Estimation Theory (New York: Academic Press).

[18] Kadison R V 1952 Ann. of Math. 56494.

[19] Bram J 1955 Duke Math. J. 2275.

[20] Conway J B 1991 The theory of Subnormal Operators, Math. Surveys and Monographs, Vol. 36 (Amer. Math. Soc.).

[21] Naïmark M A 1940 Isvest. Akad. Nauk SSSR, Ser. Mat. 453 (In Russian, with English summary).

[22] Ahkiezer N I and Glazman I M 1978 Theory of Linear Operators in Hilbert Space 3rd ed. (Vishcha Shkola) (In Russian), 1981 (Pitman) (English edition).

[23] Howe R and Tan E C 1992 Non-Abelian Harmonic Analysis (New York: Springer).

[24] Knapp A 1986 Representation Theory of Semisimple Groups (Princeton University Press).

[25] Reed M and Simon B 1980 Method of modern mathematical physics I Functional Analysis 2nd ed. (New York: Academic Press).

[26] Daubechies I 1992 Ten Lectures on Wavelets (SIAM).

[27] Sakaguchi F and Hayashi M 1999 A Relationship between the Continuous Wavelet Transformation and the Algebra $\mathrm{su}(1,1)$, Proceedings of 22nd Symposium on Information Theory and its Applications, Echigoyuzawa, Japan pp. 83.

[28] Aslaksen E and Klauder J R 1968 J. Math. Phys. 9206.

[29] Reed M and Simon B 1975 Method of modern mathematical physics II Fourier Analysis, SelfAdjointness (New York: Academic Press).

[30] Caves C M 1981 Phys. Rev. D 231693. 